\title{
A Train Protection Logic based on Topological Manifolds for Virtual Coupling
}

\author{
Yong Zhang, Haifeng Wang, Phillip James, Markus Roggenbach and Daxin Tian
}

\begin{abstract}
Virtual coupling is a promising innovation aimed at increasing railway capacity. Compared to current railway signaling systems, it allows two or more trains to run with reduced headway between them. However, such reduced headways are a challenge to safety. In this work we consider this challenge by formally describing and verifying an approach to virtual coupling. We propose a general modeling method based on topological manifolds to describe the safety protection logic for virtual coupling train control systems. We also describe the basic train control elements in topological terms and analyze the line condition of our virtual coupling logic. We establish that the line condition safety requirements and its representation as a manifold are equivalent and further provide a formal definition of the concept of a movement authority with manifold notations. This allows us to consider the dynamic behavior of trains and a series of theorems that establish the correctness of our safety protection logic for virtual coupling. Finally, we apply the presented methods to a case study. The results show that the proposed method provides a suitable way to realize a virtual coupling logic safely.
\end{abstract}

Index Terms-Virtual coupling control, Manifold based model, Train-centric train control system, Formal verification

\section{INTRODUCTION}

$\mathbf{T}$ RAIN control systems are designed to guarantee the operational safety of trains with high efficiency. The European Train Control System (ETCS) level 2 is the current state of the art for train operation and has been applied successfully in many countries. However, the railway community still faces growing demands to increase railway line capacity on existing tracks [1]. Increasing capacity is also one of the primary objectives of the Shift2Rail program, which outlines future rail-focused research and innovation activities [2]. Much

This work was supported by the China Postdoctoral Science Foundation (2021M690295), the Key Project of Chinese National Programs for Fundamental Research Development (973 program, 2014CB340703), the National Key Research and Development Program of China (2018YFB1201500), the Beijing Municipal Natural Science Foundation (L191001), the National Natural Science Foundation of China (62173012, U20A20155 and 61822101), the Newton Advanced Fellowship (62061130221), and the Science and Technology Research and Development Program of China State Railway Group Co., Ltd. (P2018X011) (Corresponding author: Haifeng Wang).

Y. Zhang is with the School of Transportation Science and Engineering, Beihang University, Beijing 100191, China (e-mail: zhangyongzhy@buaa.edu.cn).

H. Wang is with the National Engineering Research Center of Rail Transportation Operation and Control Systems, Beijing Jiaotong University, Beijing 100044, China and also with the Beijing Laboratory of Urban Rail Transit, Beijing, China (e-mail: hfwang@bjtu.edu.cn).

P. James and M. Roggenbach are with the department of computer science, Swansea University, Swansea, SA1 8EN, United Kingdom (email:p.d.james@swansea.ac.uk; m.roggenbach@swansea.ac.uk).

D. Tian is with the School of Transportation Science and Engineering, Beihang University, Beijing 100191, China (e-mail: dtian@buaa.edu.cn). exploration has been undertaken by railway researchers and institutions towards this endeavor [3]-[6]. New train-to-train communication techniques show better performance compared to current centralized communication-based train control systems [7]. Based on such train-to-train communication techniques, advanced signaling approaches such as virtual coupling can be realized to improve the utilization and efficiency of a railway line [8]. It has been shown that virtual coupling can decrease headway between trains effectively by creating multiple train convoys [9]. Specifically, in [10], [11], concrete methods based on control theory are proposed to demonstrate the performance of virtual coupling.

However, with such advancement comes skepticism with regards to level of safety, especially for safety critical railway signaling systems. Here we aim to provide a formally verified virtual coupling approach. Formal methods and formal verification provide an effective way to improve/analyze the safety of a system as suggested by EN50128 [12], especially for safety critical systems such as railway signaling systems [13]. Numerous efforts have been made to apply formal methods in railway systems [14]-[16]. $\mathrm{Vu}$ et al. presented a formal verification tool suite to verify the sequential release feature of interlocking systems [17]. Berger et al. verified the European Rail Traffic Management System (ERTMS) with real-time Maude [18] whilst James et al. have presented an extendable tool-set to verify the interlocking system combining several formal verification methods [19]. Another research issue is model-based functional testing, which could also improve the safety of the system effectively [20]. This approach has already been used for the testing of ETCS on-board controllers [21], [22].

Most of these efforts have been focused on applying general formal modeling techniques, which may not describe the train operation principles perfectly [23]. For this reason, researchers have devoted much effort to the development of domain specific languages to describe and verify railway system properties more efficiently [24], [25]. In particular, Wang et al. have proposed a point-set topology based modeling method for the current Chinese Train Control System (CTCS) level 3 standard, which reflects fixed block signaling principles through space represented by basic elements of the train control system [26]. Furthermore, Wang et al. presented a safety monitor for interlocking systems through the use of point set theory [27]. The work focused on train control systems that are essentially based around a discrete control logic. As for virtual coupling logic, the intelligent processing unit of the system should take the moving train state into consideration. In order to capture this continuous train movement, 
safety assurance researchers have explored the application of hybrid specification languages. For example, Platzer et al. applied theorem proving methods based on hybrid systems to verify aspects of ETCS based on differential dynamic logic [28]. Similarly, Zhang et al. have presented an online safety observer based on hybrid reachability analysis for the on-board equipment of CTCS-3 in which the continuous train movement is modeled by hybrid automata [29].

Physical train movement takes place on an $\mathrm{x}-\mathrm{y}$ plane with different speed (so the movement of a train can be described as a curve in a 3-dimension coordinate: 2-dimensional track layout plane and speed). Since the aim of train control systems is to separate trains as closely as possible, the control logic is not only about how to realize speed control in order to allow trains to run closer, it also considers how the relevant switches to compose the single line for trains to operate.

In traditional ETCS-like systems, those functions are dispatched to different subsystems to be fulfilled. For example, the route control process is usually done by an Interlocking system, the movement authority is dispatched to a Radio Block Center (RBC), and the over-speeding protection function is fulfilled by on-board equipment. While for train-centric control systems, these functions are undertaken only by the on-board equipment. Consequently, there is a need to study effective modeling methods for both control systems to implement somewhat complex virtual coupling logic.

Normal arithmetic is used in the speed control function of current train control system, but is not suitable for the switch control process. The method mentioned in reference [26] only considers the line resources arrangement in the railway line but does not take the speed control process into consideration, which is a continuous behavior of trains. So, we adopt the presented manifold theory to model the train behavior under virtual coupling control. With the model described by the manifold, we can analyze, model, and deduce the whole control process including switch control, train order, and overspeeding protection modules under the same mathematical framework.

Virtual coupling in a train-centric way has also been considered in [30] who also use concepts of topological manifolds. The main difference is the work only focused on traincentric control systems. In particular it provided algorithms for monitoring the virtual coupling logic where the monitor would interfere should non-safe situation arise according to the behavior of trains. While for this paper, we address virtual coupling on a fundamental level, which is independent of the control system. Our approach works for both traincentric (distributed) or current state of the art ETCS/CTCS (centralized) control systems. We provide new topological concepts to develop new safety conditions. Also the effect of switches on virtual coupling logic has been analyzed. A map function is proposed to tackle the relevant location relation between dynamic train location and static basic equipment locations. Using the notion of manifolds, the control of the switches (movements in the track layout plane) and the speed adjustment (movement in track layout-speed space) have been modeled in a unified methodology.

In addition, the conditions for which trains can be virtually coupled are clarified based on a topological representation of train control elements through proving the equivalence between the introduced manifold condition and the safety requirements needed for virtual coupling to occur. Movement authority is re-defined by a notion based on manifolds, which takes the dynamic behavior of trains into consideration. A worst-case condition is considered in order to make the dynamic behavior closer to reality. Train length is also taken into consideration in the definitions of manifold curves. Furthermore, a series of theorems are presented that prove the safety of our proposed computation method. That is, based upon extending the notion of space (from traditional distance to distance-speed), the principle of separating spaces for different trains is preserved by the manifold based movement authority definition.

Our methodology models concrete track sections, switches, trains within the train control domain (which are presented in a traditional view) as abstract topological elements (which are presented in a topological view), which leads to a rigorous unified formal model. The model also reflects the property of train control principles in a natural way, beyond specific basic railway equipment (switches etc.). As a benefit of its mathematic nature, one can better understand the whole integrated train control concept and reuse the manifold theorems to prove its safety. The model also contributes to a less error-prone development of a virtual coupling system implementation, due to its unambiguous description and proved safety guarantee.

Since the notion of a "point" appears in both railway systems and topological manifold theory, we will use the American term "switch" in place of the British term "point" for the railway component in order to avoid confusion with the point in topological manifold theory.

The rest of this paper is organized as follows. In section II, we introduce the details of virtual coupling. Section III gives the theory of our manifold modeling methods for virtual coupling. A case study is then given in section IV to illustrate our method. Finally, section V concludes the paper by outlining future aspirations and directions.

\section{VIRTUAL COUPLING}

We now introduce virtual train coupling, basing on the definition of [30], which however was thought to be implemented as an independent module of the on-board equipment, in a train-centric control system. However, train-to-train communication is required to implement virtual coupling in both systems. In this paper, we assume that wireless train-to-train communication has no time delays. As for the implementation in ETCS like systems, the general model of the protection logic would rely on existing basic equipment, and the safety effect led by basic equipment locations would be further analyzed.

In this section, we first compare train movements under moving block with those under virtual coupling. Then we come up with two questions about the implementation of virtual coupling and analyze the safety line conditions under virtual coupling logic on the basis of existing basic equipment, which was not considered in [30]. 

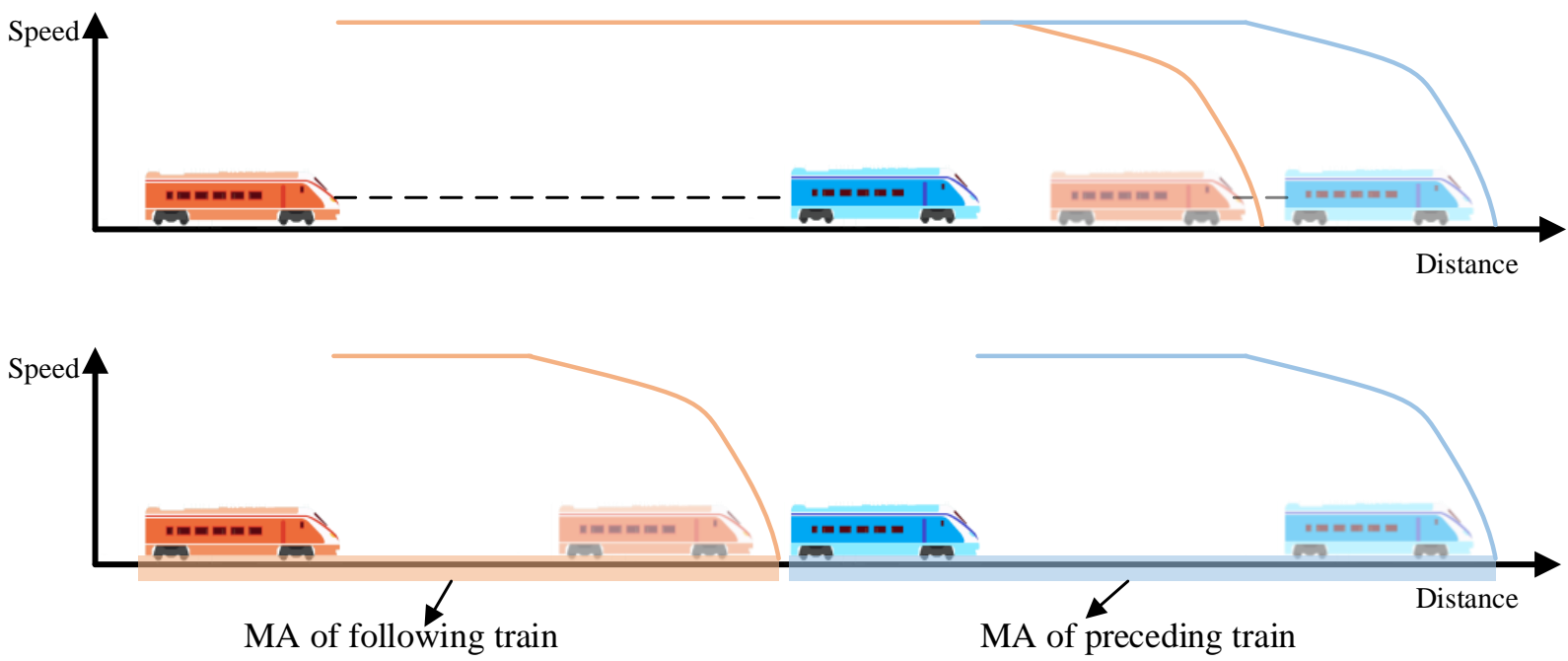

Fig. 1: Comparison between virtual coupling (top) and moving block (bottom) control logic

\section{A. Basic principles of Virtual coupling}

Virtual coupling is an advanced signaling control concept that greatly improves capacity with respect to traditional fixed block approaches [30]. Trains running under a virtual coupling scheme with the same travel direction will be allowed to run closer than the trains using traditional fixed/moving block strategies. Actually, it is also considered superior to moving block systems, however has yet to be implemented.

The difference between moving block and a virtual coupling logic is shown in Fig. 1. For two trains operating in the same direction as shown in Fig. 1, the following train is the orange train on the left, and the preceding train is the blue one on the right. The semi-transparent trains represent the location trains could reach in the future. For the moving block systems (the bottom picture in Fig. 1), the maximal End of Authority (EoA, the location to which the train is authorized to move and where the target speed is zero.) of the following train would be the current location of the preceding train. For the virtual coupling logic (the top picture in Fig. 1, the dashed line represents the trains are virtually coupled), the final stop location of the following train exceeds the location where the preceding train currently is.

Virtual coupling has been shown to increase capacity, cf., e.g., [31]. The headway for scenarios frequently seen in real world could be decreased by $43 \%$ compared to ETCS level 3 system on which moving block is adopted. Without considering all the different scenarios where virtual coupling leads to capacity gains, we want to point out that as shown in Fig 1, the separation distance between the semitransparent trains is much smaller in the coupled train scenario than in moving block scenario. Virtual coupling relies on the assumption that the preceding train will not come to a stop instantly. Furthermore, the brake operation of the lead train is known to the following train thanks to train-to-train communication.

In order to realize virtual coupling, there are two basic problems that need to be settled.

1) The first problem deals with how to insert or remove a train from the virtual coupling queue. The destinations of different trains vary according to their own scheduled plans. However, they may share a common railway line in a specific area during their whole travel. How to insert/remove a train into a queue when a following train enters/leaves the shared line is a critical question of virtual coupling control.

2) The second problem is the movement authority computation logic for trains in a queue, which considers various over-speeding protection functions. The movement authority computation should take the movement of both the preceding train and following train into consideration, which is vastly different to the current computation processes.

\section{B. Safety analysis of railway line conditions under virtual coupling}

In order to realize the virtual coupling logic, the accurate speed and location of trains should be taken into consideration in the control logic for both train-centric or current train control systems. In addition, the position and locking state of switches is also essential in the control logic.

The first problem mentioned in the above section is to determine the condition for a train to virtually couple with the preceding train. The safety requirements in this situation should look at the switch positions and locking states. The distance between two trains, and between the trains and switches should also be taken into consideration. We analyze several basic scenarios to illustrate the condition for a train to be virtually coupled as shown in Fig. 2 .

In these scenarios, there are two trains named $t 1$ and $t 2$. The question is whether the following train $t 1$ could be virtually coupled with the preceding train $t 2$. The destinations are denoted by $D 1$ and $D 2$ for $t 1$ and $t 2$ respectively. There is one switch equipment denoted by $p 1$ in the railway line.

Firstly we will discuss the situation where the trains have different destinations.

In scenario 1 as shown in Fig. 2a, $t 1$ and $t 2$ are running in the section between stations and are far away from the 
switch contained in the station. The dashed line represents the destination paths of both trains. Even though the destinations are different, $t 1$ can still be virtually coupled with $t 2$ to gain extra capacity due to a common long overall path. When $t 2$ is approaching $p 1$ in the station as shown in scenario 2 (Fig. $2 b$ ), if the distance $d$ between $t 2$ and the switch $p 1$ is large enough for $t 2$ to brake before $p 1$, then $t 1$ can be virtually coupled with $t 2$. As for the time of switch movement, an extra distance between trains should be added by decreasing $t 1$ speed. When $t 2$ has already passed $p 1$ or the distance $d$ is smaller than the $t 2^{\prime} s$ braking distance to $p 1$, then whether $t 1$ should or should not be virtually coupled to $t 2$ would depend on the required states and current states of switch 1 as shown in scenario 2 and 3 (Fig. 2b and Fig. 2c). If the required position is not consistent with the current switch position (which is consistent with the required position by $t 2$ ), then $t 1$ should not be virtually coupled to $t 2$. If $t 1$ is virtually coupled with $t 2$ under this situation, $p 1$ might not be moved to the right position required by $t 1$ in time, which would lead to derailment of $t 1$.

Now we discuss the situation in which the trains travel to the same destination, which means both $t 1$ and $t 2$ will pass $p 1$ as scheduled with the same position. In this case, whether or not trains are approaching the switch would not be a problem, the only concern being that the specific relevant switches should be locked into the right position as required by the two trains. As a result, we just draw one picture in which train $t 2$ passed switch $p 1$ as shown in scenario 4 (Fig. 2d).

The line condition for trains safely running under virtual coupling is that there should be an overlapped path between the destination path and the train-to-train path, and if switches are included in the overlapped path or the distance $d$ as introduced in the scenario above, the switch should be locked in the right position for trains to pass.

Here the switch $p 1$ could be only one switch, or a complex composition of several switches. Since the train will pass the switches one by one, this composition can be considered as a sequence of switches. If the required position of any switch in the sequence is the same, then this switch could be treated as a part of the straight railway line. If the required position of any is different, then it comes to the situation introduced above. In that case, complex switch compositions could be tackled in the same way as described above.

The second problem would be how to realize the safety protection of virtual coupling control regarding the location and speed of trains once the railway line condition analyzed in section II-B is satisfied. Traditional railway signaling technology would divide the whole control process into several functions, e.g., the MA computation and the speed control process. Each function is undertaken by specific equipment. The MA is actually a discrete allocation logical computation result described by the track sections in the traditional signaling system. The track sections allocated give a separate space for each train to operate without the possibility of collision.

In virtual coupling control systems, trains run closer than under traditional fixed/moving block principles. As a result, the new "MA" information should consider train speed and location information, and also obey the train separation concept. In

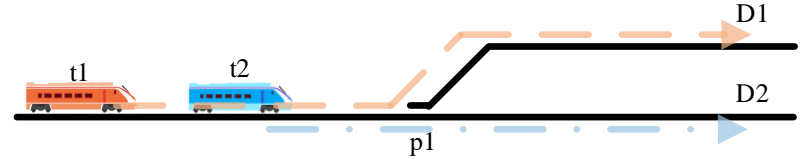

(a) Scenario 1: trains (t1 and $\mathrm{t} 2$ ) are far away from the switch $p 1$

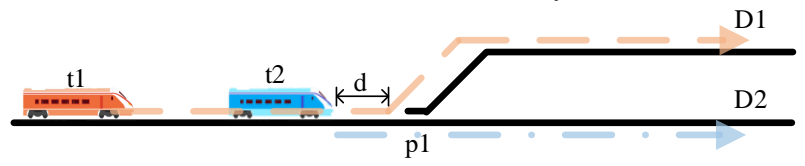

(b) Scenario 2: preceding train (t2) are approaching switch $p 1$

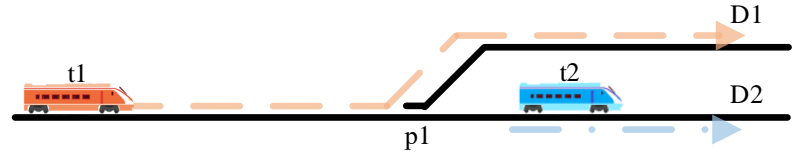

(c) Scenario 3: preceding train (t2) has passed switch $p 1$

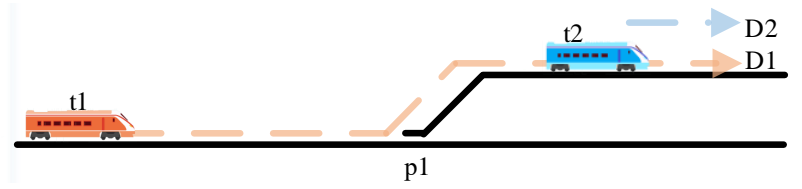

(d) Scenario 4: both trains share the common elements

Fig. 2: Illustration of the line condition under different scenarios on virtual coupling logic

order to guarantee the safety of train operation, a correct MA should be allocated to each of the trains, which ensures that the movement inside the MA does not lead to collisions with the obstacles in front of trains. The movement of a train should be controlled inside the allocated space during operation to avoid possible collisions. Lastly, the allocated MA of different trains should not overlap.

\section{MANIFOLD BASED TRAIN CONTROL}

In this section, the problems mentioned in section II-A will be described based on topological manifold theory. Traditionally, railway systems and the features of the elements are described using natural language. Often these descriptions are very detailed and lengthy, describing all the characteristics of the railway line elements. This often leads to engineers or developers paying attention to the information of specific tracks or switches, but makes it easy to ignore the topological relations among basic equipment, which represent the nature of railway signaling principles.

Here we adopt the topological view on the railway elements as a kind of abstraction to implement the virtual coupling logic in a mathematical based format. Based on that we could describe the safety protection logic rigorously and better address the core railway signaling principles. A topological description of the CTCS-3 system has been proposed in [26], [27], [32]. All the previous work is about discrete representation without considering the speed and location of trains. We therefore adopt the topological manifold concept to describe the movements of trains on the railway line.

Compared with our previous work, several new definitions are introduced for describing elements or used in the proof of theorems. Track sections and trains are also described as 
topological elements. Hence, the proposed modeling method could be used in current centralized train control systems. In order to formalize the virtual coupling line condition a path notion is proposed to help describe the condition, and also the intersection of two paths is introduced. In addition, a sequence map function is proposed to determine the relevant location relation among topological elements. As for the movement authority under virtual coupling, we define several curves to describe different train movements. These differ from the curves proposed in our previous work, we consider train behavior under worst conditions, which make the movement authority more suitable to the accurate virtual coupling requirement. The definitions of the curves also take train lengths into consideration, which is closer to reality. At the end of this section, three theorems are proved to clarify the safety of the virtual coupling logic.

\section{A. Preliminaries}

Definition 1: A topology on a set $S$ is a collection $\mathcal{T}$ of subsets containing both the empty set $\emptyset$ and the set $S$ such that $\mathcal{T}$ is closed under arbitrary unions and finite intersections. The pair $(S, \mathcal{T})$ is called a topological space [33].

To simplify the notation, we refer to a pair $(S, \mathcal{T})$ as the "topological space $S$ " in the rest of the paper. Hence, when we refer to a subset or to the cardinality of a topological space $(S, \mathcal{T})$, we refer to a subset of $S$, or to the cardinality of $S$, hence freely using notations $X \subseteq S$, or $|S|$.

Definition 2: An open ball $B(p, r)$ with center $p \in \mathbb{R}^{n}$ and radius $r>0$ is the set $B(p, r)=\left\{x \in \mathbb{R}^{n} \mid d(x, p)<r\right\}$, where $d(x, p)$ is the distance between two points $x$ and $p$ in Euclidean space of $n$ dimension $\mathbb{R}^{n}$ as shown in equation 1 .

$$
d(x, p)=\left[\sum_{n}^{i=1}\left(x_{i}-p_{i}\right)^{2}\right]^{1 / 2}
$$

For simplicity, we denoted $B(p, r)$ as $B, B^{n}$ represents an open ball of dimension $\mathrm{n}$.

Definition 3: If $X$ and $Y$ are topological spaces, a homeomorphism from $X$ to $Y$ is a bijective map $\phi: X \rightarrow Y$ such that both $\phi$ and $\phi^{-1}$ are continuous. If there exists an homeomorphism between $X$ and $Y$, we say that $X$ and $Y$ are homeomorphic [33].

Definition 4: Given a point $p$ in a topological space $X$, a neighborhood of $p$ is a subset $V$ of $X$ that includes an open subset $U$ containing p, i.e., $p \in U \subseteq V$.

Definition 5: A topological space $M$ is locally Euclidean of dimension $n$ if every point $p$ in $M$ has a neighborhood $U$ such that there is homeomorphism $\phi$ from $U$ onto an open subset of $\mathbb{R}^{n}$. We call the pair $\left(U, \phi: U \rightarrow \mathbb{R}^{n}\right)$ a chart [33], $U$ a coordinate neighborhood and $\phi$ a coordinate map on $U$.

Definition 6: A topological space $X$ is said to be a Hausdorff space if given any pair of distinct points $p_{1}, p_{2} \in X$, there exist neighborhoods $U_{1}$ of $p_{1}$ and $U_{2}$ and $p_{2}$ with $U_{1} \cap U_{2}=\emptyset$ [34].

Definition 7: A topological space $X$ is said to be second countable if it admits a countable basis for its topology $\mathcal{T}$. A countable sub-collection $\mathcal{B}$ of $\mathcal{T}$ is a countable basis for the topology $\mathcal{T}$, if given an open set $U$ and point $p$ in $U$ there is an open set $B \in \mathcal{B}$ such that $p \in B \subset U$ [33].
Definition 8: A $n$-dimensional topological manifold $M$ is a second countable Hausdorff space that is locally Euclidean of dimension $n$ [34].

Loosely speaking, a manifold is a topological space that is locally Euclidean. It is a generalization of curves and surfaces to higher dimensions. The coordinates on a chart allow us to carry out computations as though in an Euclidean space.

Definition 9: A function is $C^{\infty}$ means that the function is differentiable for all degrees of differentiation [33].

Definition 10: Two charts $\left(U, \phi: U \rightarrow \mathbb{R}^{n}\right)$ and $(V, \psi: V \rightarrow$ $\mathbb{R}^{n}$ ) of a topological manifold are $C^{\infty}$ compatible if the two maps

$$
\begin{gathered}
\phi \circ \psi^{-1}: \psi(U \cap V) \rightarrow \phi(U \cap V), \\
\psi \circ \phi^{-1}: \phi(U \cap V) \rightarrow \psi(U \cap V)
\end{gathered}
$$

are $C^{\infty}[33]$.

Definition 11: A $C^{\infty}$ atlas on a locally Euclidean space $M$ is a collection $\mathcal{U}=\left\{\left(U_{\alpha}, \phi_{\alpha}\right)\right\}$ of pairwise compatible charts that cover $M$, i.e., such that $M=\bigcup_{\alpha} U_{\alpha}$ [33].

Definition 12: A n-dimensional manifold with boundary is a second countable Hausdorff space in which every point has neighborhood homeomorphism either to an open subset of $\mathbb{R}^{n}$, or to an open subset of $\mathbb{H}^{n}$ [33], where $\mathbb{H}^{n} \subset \mathbb{R}^{n}$ is defined by equation 3 .

$$
\mathbb{H}^{n}=\left\{\left(x_{1}, \ldots x_{n}\right) \in \mathbb{R}: x_{n} \geq 0\right\}
$$

Definition 13: If $\mathcal{M}$ is an n-manifold with boundary, a point $p \in M$ is called an interior point of $\mathcal{M}$ if it is in the domain of an interior chart; and it is called a boundary point of $\mathcal{M}$ if it is in the domain of a boundary chart that takes $p$ to $\mathbb{H}_{n}$. The boundary of $\mathcal{M}$, denoted by $\partial \mathcal{M}$, is the set of all its boundary points, and its interior, denoted by $\operatorname{Int} \mathcal{M}$, is the set of all its interior points [34].

\section{B. Basic elements representations}

The virtual coupling system could be implemented on the basis of current ETCS systems or newly proposed train-centric control systems. Since a train operates on a railway line, the basic elements of the railway line need to be defined. As for current CTCS-3 or ETCS-2 systems, signals are not needed for the control process. For future ETCS-3 or train-centric control systems, track sections are also eliminated. However, in order to be compatible with as many as possible train control systems, in this paper, we discuss the virtual coupling model with general basic equipment, which are track sections, switches and signals (which are mentioned as stop locations for trains in this paper for the sake of virtual coupling logic).

In addition, a train is defined to be a topological point in the computation of the protection logic. In what follows we introduce definitions of basic elements in current train control systems.

Definition 14: A train stop location is represented by a triple $s=(p$, dir, res $)$, where

- $p$ is the stop location in $(\mathrm{x}, \mathrm{y})$ coordinates,

- dir is the direction the train travels in,

- res is the reserved state of the stop location. 
There are only two directions for a train to travel, dir $=1$ means that the train travels in the down direction, and $\operatorname{dir}=0$ for the up direction.

The reserved state of a stop location indicates if the stop location is reserved for a specific train to pass or not. If the stop location is reserved by no train, res $=0$. If it is reserved by one train, then res $=1$.

We define track sections without switches inside them.

Definition 15: A track section without a switch is a connected non-empty set (intuitively a line segment) and represented by a 6-tuple $t s=(p l, p r, d i r, o s, l s, r e s)$, where

- $p l$ is the left end location in (x,y) coordinates,

- $p r$ is the right end location in $(\mathrm{x}, \mathrm{y})$ coordinates,

- dir is the direction of the track section when train passes,

- os is the occupation state of the track section,

- $r s$ is the required occupation state of the track section for virtual coupling,

- $l s$ is the locking state of the track section,

- res is the reserved state of the track section.

$p l$ and $p r$ are location values of the left and right end point of the segment. dir has the same meaning as it is in the definition of stop location. $o s=0$ represents the track section is occupied and $o s=1$ unoccupied. The value of $r s$ represents the required state of the track section when it is used for virtual coupling logic of trains. $r s=0$ represents the track section is connected from $p l(p r)$ to $p r(p l)$ and also unoccupied by a train, $r s=1$ represents the track section is unconnected and occupied by a train. The value of $l s$ represents the locking state of the track section, $l s=1$ represents that the track section is locked, and $l s=0$ represents that it is not locked. The value of res also has the same meaning as explained in the stop location definition above.

We define a function $\Lambda_{T}(t s)$ in order to know whether a track section can be used to generate the movement authority under virtual coupling logic.

The function $\Lambda_{T}(t s)$ can be computed according to the information of track section $t s$ as shown in equation 4. If $\Lambda_{T}(t s)=1$, it means that the track section $t s$ is safe to generate the movement authority. If $\Lambda_{T}(t s)=0$, the track section $t s$ is not allowed to generate the movement authority due to safety issues. Here, we assume that the track sections would work as designed, i.e., if there is a train on the track, then the track reflects an occupied state.

$$
\Lambda_{T}(t s)=t s . l s
$$

Another basic element of a railway line for train control systems are switches. A switch is represented by a 9-tuple as shown in definition 16. It is also a connected non-empty set and the stem location denoted as $\mathrm{pm}$ is in the middle of the line segment intuitively, as shown in Fig. 3. The $p n$ and $p r$ are the normal end location and reverse end location of the switch. The normal position of a switch would allow train travel from $p m$ to $p n$ or from $p n$ to $p m$, the reverse position would allow train travel from $p m$ to $p r$ or from $p r$ to $p m$. The value of $d s$ represents the position of a switch, $d s=0$ represents that the switch is in normal, $d s=1$ represents that the switch is in reverse position, and $d s=2$ represents the switch has lost
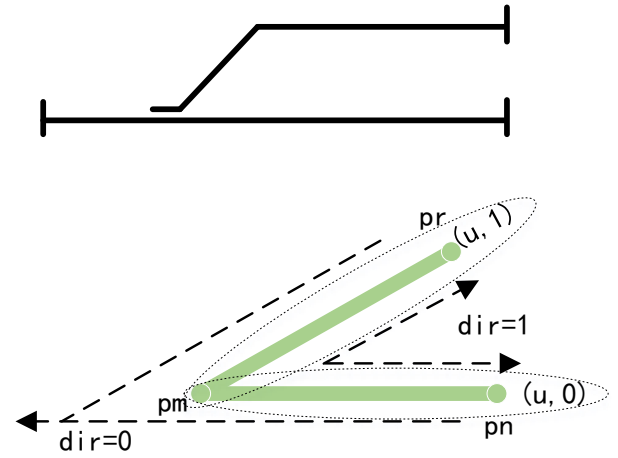

Fig. 3: The switch unit of the railway line

indication. The value of $l s$ has the same meaning as defined in 15 .

The reserved state of the switch indicates if the switch is reserved for a specific train to pass or not. The value of res is the id of the train which reserves the given switch. If the given switch is not reserved by any train, res $=0$. A switch can only be reserved by one train at a time. Once the switch is reserved, it cannot be controlled by commands from other trains.

The value of $r s$ represents the required switch position for a path, $r s=0$ represents that the required switch position is in normal and the switch is connected from $p m(p n)$ to $p n$ $(p m), r s=1$ represents that the required switch position is in reverse and the switch is connected from $p m$ ( $p r)$ to $p r$ $(\mathrm{pm})$, and $r s=2$ represents that the path does not need this switch at any specific position and the switch is not connected from $p m$ to either $p r$ or $p n . r s=3$ represents that the required switch position is unclear, i.e. the required position is different for this switch by different paths.

Definition 16: A switch of a railway line is represented by a 9-tuple $p t=(i d, p m, p n, p r, d i r, d s, r s, l s, r e s)$, where

- $i d$ is the identification of the switch in the railway line,

- $p m$ is the stem location of the switch in $(\mathrm{x}, \mathrm{y})$ coordinates,

- $p n$ is the normal end location of the switch in $(\mathrm{x}, \mathrm{y})$ coordinates,

- $p r$ is the reverse end location of the switch in $(\mathrm{x}, \mathrm{y})$ coordinates,

- $d i r$ is the direction of the switch when train passes,

- $d s$ is the position of the switch,

- $r s$ is the required switch position by a path through it,

- $l s$ is the locking state of the switch,

- res is the reserved state of the switch.

Among the attributes of a switch defined in definition 16, some are static, given by the physical location of the switch, while some are dynamic and express the state of the switch.

For a switch $p t$, we define a function $\Lambda_{P}(p t)$ to know whether it can be used to generate a movement authority under virtual coupling logic. The function $\Lambda_{P}(p t)$ can be computed according to the information of switch $p t$ as shown in equation 5. If $\Lambda_{P}(p t)=1$, it means that the switch $p t$ is safe to generate the movement authority. If $\Lambda_{P}(p t)=0$, the switch $p t$ is not allowed to generate the movement authority due to safety issues. 


$$
\Lambda_{P}(p t)=\left\{\begin{array}{lll}
1, & \text { if } & p t . d s=p t . r s \text { and pt.ls }=1 \\
0, & \text { if } p t . l s=0, \text { or } p t . d s \neq p t . r s .
\end{array}\right.
$$

The topological representation of a train is defined as shown in definition 17. The stop location of a train is calculated by the safe brake curve (to be introduced in section III-D), which is used to determine the sequence of basic elements in the topological representation of the railway line.

Definition 17: A train in a railway line is represented by a 7-tuple $t r=(i d, p s, p e, p r, v, d i r, d e c)$, where

- $i d$ is the identification of the train in the railway line,

- $p s$ is the current location of train's maximum safe front end in (x,y) coordinates,

- $p e$ is the final stop location of maximum safe front end $\xi_{s}(s, 0)$ in $(\mathrm{x}, \mathrm{y})$ coordinates of the train following the safe brake curve,

- $p r$ is the current location of train's minimum safe rear end in $(\mathrm{x}, \mathrm{y})$ coordinates,

- $v$ is the current speed of the train,

- dir is the direction of the train traveling,

- dec represents the emergency brake deceleration of the train.

In our model, we consider locations of a train's maximum safe front end and minimum safe rear end for the sake of inaccuracy of train positioning functions. We assume that those locations could be obtained by considering train length and error margins of specific train positioning equipment.

The traditional view shows the actual location of trains and switches on the railway line. The topological view is how the physical location is mapped into a topological representation with a sequence. Both have enough information for the logic computation. The beneficial thing is that in the topological representation, we could focus on the topological feature (compactness, connectivity etc.) of railway elements on an abstract level when we need, without considering specific locations, lengths and states accordingly. If we need the specific information, we still could acquire them through the attributes of topological elements to fulfill the calculation.

The switch and stop location elements can be transferred into topological representations according to the railway line layout. Since static elements like switch and stop location are fixed, their order in the topological representation is also fixed. However the location of a train changes along with the movement of the train. The train could therefore be mapped into the topological representation according to the dynamic speed and location of the train. If the train $t$ is located near a switch $p t$, a track section $t s$ or stop location element $s$, then the sequence of the topological representation between a train and other elements could be determined by the function $\mathcal{H}(t, *)$, where $*$ could be either $p t, t s$ or $s$, and $*$.p represents any of the location parameters of the switch or stop location.

The function $\mathcal{H}(t, *)$ takes the train $t$ and the near railway element (track section, switch or stop location) as input, then outputs relative position of $t$ and the near element (whether a train is in front of the near railway element or otherwise).

We define that the location value of railway elements is growing from left to right, i.e., for two railway elements $*_{1}$,
$*_{2}$, if $*_{1} . p<*_{2} . p$, then $*_{1}$ is located on the left of $*_{2}$. Consequently, the map function $\mathcal{H}(t, *)$ could be determined as shown in equation 6 .

$$
\mathcal{H}(t, *)= \begin{cases}\langle *, t\rangle, & \text { if } * . p<\min (t . p r, t . p e) \text { or } \\ & \text { t.dir }=1, t . p r<* . p<t . p e \\ \langle t, *\rangle, & \text { if } * . p>\max (t . p r, t . p e) \text { or } \\ & \text { t.dir }=0, \text { t.pe }<* . p<t . p r\end{cases}
$$

For example, train $t$ is running from the left to right ( $t$.dir $=$ 1) as shown in Fig. 4. In 4a, $t$ is located far from the switch $p t$, i.e. t.pr $<$ pt.pm, t.pe $<$ pt.pm. As a result, the elements in topological view would be described as shown in Fig. 4a.

When train $t$ continues running until t.pr $<p t . p m<t$.pe as shown in Fig. 4b, we could map the sequence of $t$ and $p t$ through $\mathcal{H}(t, p t)$ as shown in topological view of Fig. $4 \mathrm{~b}$. Compared to the sequence of Fig. $4 \mathrm{~b}$, the relation of $t$ and $p t$ are still neighbors but with a different sequence.

Train $t$ needs to take the state of switch $p t$ into consideration in order to ensure safety of train operation. When the minimum rear end location of train $t$ has passed the switch, the elements in the topological view are shown in Fig. 4c.

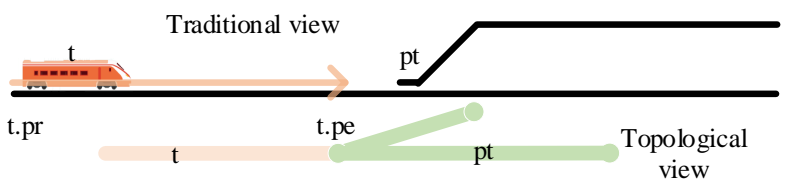

(a) Scenario 1: train $t$ is located on the left of the switch $p t$

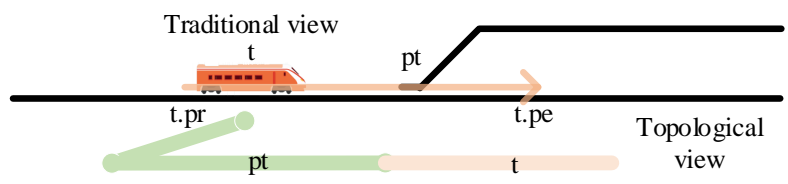

(b) Scenario 2: train $t$ are approaching switch $p t$

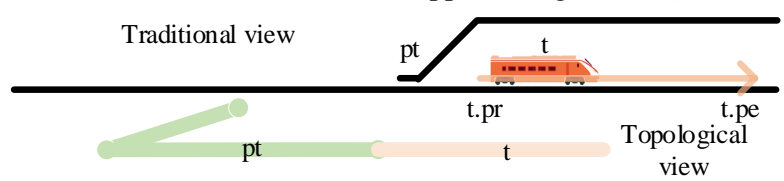

(c) Scenario 3: train $t$ has passed switch $p t$

Fig. 4: Illustration of the train element map to the topological representation

\section{Line condition on virtual coupling}

In order to realize the virtual coupling control there is a limit condition for the railway line elements, which could affect the safety of train operation as described in section II-B.

Based on the basic topological elements above, we firstly define some basic notations which could help further represent the train trajectory on the railway line.

We define $U$ to represent the specific topological element of the railway line, which could be denoted as a pair as shown in equation 7. $u$ and $u . r s$ represents the abstract shape of corresponding topological elements. For example, if $u_{1}$ represents a specific switch, then $u_{1}$ is the shape of a whole with two directions to go when we mention $u_{1}$, and $u_{1}$.rs 


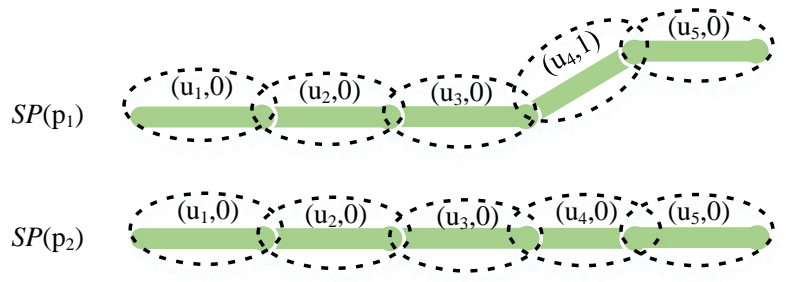

Fig. 5: Illustration of the Topological spaces of two paths

represents just one direction of the switch (segment between $u_{1}$.pm and u1.pn or between $u 1 . p m$ and $\left.u 1 . p r\right)$. As for train and stop location, we could represent them only by $u$, i.e., if $u$ represents a train or stop location, then $u . r s=N U L L$.

$$
U=(u, u . r s)
$$

Then, we describe a specific railway element intuitively with a pair $\gamma_{i}$ :

$$
\gamma_{i}=\left(u_{i}, u_{i} \cdot r s\right)
$$

In order to clarify topological manifold property of switches or track sections regarding their position or occupation state, we define two projection functions to map the topological element to specific states as shown in equation 9. $\operatorname{Pro}_{1}\left(\gamma_{i}\right)$ and $\mathrm{Pro}_{2}\left(\gamma_{i}\right)$ map $\left(u_{i}, u_{i} . r s\right)$ onto $u_{i}$ or $u_{i}$.rs accordingly.

$$
\begin{array}{r}
\operatorname{Pro}_{1}\left(\gamma_{i}\right)=\left\{u_{i} \mid \exists \gamma_{i} \in p, u_{i}=\operatorname{Pro}_{1}\left(\gamma_{i}\right)\right\} \\
\operatorname{Pro}_{2}\left(\gamma_{i}\right)=\left\{u_{i} . r s \mid \exists \gamma_{i} \in p, u_{i} . r s=\operatorname{Pro}_{2}\left(\gamma_{i}\right)\right\}
\end{array}
$$

We then define the notion of path as:

Definition 18: A path is a set of connected topological elements which is denoted as a sequence of topological elements pair:

$$
p=\left\langle\gamma_{0}, \gamma_{1}, \ldots, \gamma_{n}\right\rangle
$$

The topological space of a path is a power set of the points in every topological elements, which could be imagined as segments as shown in Fig. 5.

We denote the topological space of a path $p$ as $S P(p)$. The projection of a path $p$ could be obtained by calculating the corresponding projection function $\left(\mathrm{PrO}_{1}\right.$ or $\left.\mathrm{Pro}_{2}\right)$ of every element $\gamma_{i}$ in $p$, which is denoted by $\left.\operatorname{Pro}_{(} p\right)$ as shown in equation 10 . The suffixes 1 or 2 could represent specific projection function accordingly.

$$
\operatorname{Pro}(p)=\left\{\left\langle u_{0}, u_{1}, \ldots, u_{n}\right\rangle \mid \forall \gamma_{i} \in p, u_{i}=\operatorname{Pro}\left(\gamma_{i}\right)\right\}
$$

In this paper, we consider two special kinds of paths which are analyzed in section II-B:

1) A destination path starting from a train to its scheduled stop location is represented by $p_{T L}=\left\langle\left(u_{0}, u_{0} . r s\right),\left(u_{1}, u_{1} . r s\right), \ldots,\left(u_{n}, u_{n} . r s\right)\right\rangle$, where $\left(u_{0}, u_{0} . r s\right),\left(u_{1}, u_{1} . r s\right), \ldots,\left(u_{n}, u_{n} . r s\right) \quad$ are the basic topological elements from the train to the scheduled stop location of the railway network. The following train is denoted as $\left(u_{0}, u_{0} . r s\right)$ and the scheduled stop location is denoted as $\left(u_{n}, u_{n} . r s\right)$. We denote the topological space of $p_{T L}$ as $S P\left(p_{T L}\right)$.

2) A train path starting from a train to its preceding train is represented by $p_{T T}=$ $\left\langle\left(u_{0}, u_{0} . r s\right),\left(u_{1}, u_{1} . r s\right), \ldots,\left(u_{m}, u_{m} . r s\right)\right\rangle, \quad$ where $\left(u_{0}, u_{0} . r s\right),\left(u_{1}, u_{1} . r s\right), \ldots,\left(u_{m}, u_{m} . r s\right)$ are the basic topological elements from the train to the preceding train of the railway network. $\left(u_{0}, u_{0} . r s\right)$ has the same denotation as above. The preceding train is denoted as $\left(u_{m}, u_{m} . r s\right)$. The topological space of $p_{T T}$ is $S P\left(p_{T T}\right)$.

In order to introduce conditions on this virtual coupling logic, we define the intersection set between $p_{T L}$ and $p_{T T}$ by equation 11 and denote its topological space as $S$.

$$
\begin{aligned}
& p_{T L} \wedge p_{T T}=\left\{\left\langle\gamma_{0}, \gamma_{1}, \ldots, \gamma_{n}\right\rangle \mid\right. \\
& \left.\operatorname{Pro}_{1}\left(\gamma_{i}\right) \in \operatorname{Pro}_{1}\left(p_{T L}\right) \cap \operatorname{Pro}_{1}\left(p_{T T}\right)\right\}
\end{aligned}
$$

Then we need to decide the concrete projection values for the topological element pair in the intersection set between $p_{T L}$ and $p_{T T}$. As for the shared topological element pair $\gamma_{i} \in p_{T L} \wedge p_{T T}$, the value of $\operatorname{Pro}_{1}\left(\gamma_{i}\right)$ could be obtained by $\operatorname{Pro}_{1}\left(\gamma_{i}^{\prime}\right)$ or $\operatorname{Pro}_{1}\left(\gamma_{i}^{\prime \prime}\right)$, which refer to the same railway element $u_{i}$, i.e., $\operatorname{Pro}_{1}\left(\gamma_{i}^{\prime}\right)=\operatorname{Pro}_{1}\left(\gamma_{i}^{\prime \prime}\right)=u_{i} \cdot \gamma_{i}^{\prime} \in p_{T T}$ and $\gamma_{i}^{\prime \prime} \in p_{T L}$ represent topological element pairs in two paths $p_{T T}$ and $p_{T L}$. The value of $\operatorname{Pro}_{2}\left(\gamma_{i}\right)$ could be determined by equation 12 .

$$
\operatorname{Pro}_{2}\left(\gamma_{i}\right)=\left\{\begin{array}{cll}
\operatorname{Pro}_{2}\left(\gamma_{i}^{\prime}\right), & \text { if } & \operatorname{Pro}_{2}\left(\gamma_{i}^{\prime}\right)=\operatorname{Pro}_{2}\left(\gamma_{i}^{\prime \prime}\right) \\
3, & \text { if } \quad \operatorname{Pro}_{2}\left(\gamma_{i}^{\prime}\right) \neq \operatorname{Pro}_{2}\left(\gamma_{i}^{\prime \prime}\right) .
\end{array}\right.
$$

Since the topological space is constructed by considering the safe brake distance of the train, the switch states would be considered if the distance is not large enough for the train to pass (the topological switch element would be located in front of the train as shown in Fig. 4b). In the traditional view, the safety requirements of the line condition of whether a train could be virtually coupled or not are shown in definition 19 .

Definition 19: The safety requirements of the line condition of virtual coupling is that $|S| \geq 1$ and $\forall p t \in S$,pt.ls = $1, p t . r s=p t . d s, \forall t s \in S, t s . l s=1$.

Here $|S| \geq 1$ represents that there is at least one topological element (the train itself) included in topological space $S$ of $S P\left(p_{T L}\right) \wedge S P\left(p_{T T}\right)$.

Definition 20: In the topological view, the following train could be virtually coupled with the preceding train only if the topological space $\left(S, \mathcal{T}_{S}\right)$ is a 1 -manifold and $\forall p t \in$ $S, \Lambda_{P}(p t)=1, \forall t s \in S, \Lambda_{T}(t s)=1$.

The traditional and topological views are different representations of the same objects (switches, trains etc.). The locking states and position information are essential for switches. Both views should keep this logic computation related information. The difference is that based on the topological elements, we can further analyze the implicit topological property among the specific railway equipment (which are linked by train control conditions or principles etc) and express it with the help of abstract mathematical theory. 


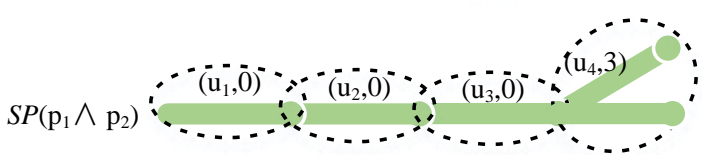

Fig. 6: Illustration of the topological space of the intersection of two paths

The theorem below proves that the condition proposed as shown in definition 20 is equivalent to the safety requirements are satisfied.

Theorem 1: A following train can be virtually coupled to the preceding train if and only if the safety requirements are satisfied.

Proof 1: The position, occupation and locking state condition of a switch and a track section is actually the same in both the topological view and the traditional view. In what follows we prove the rest of the condition.

If $|S| \geq 1$, then there is an overlapped connected path between the train-to-train path and the destination path. If no switches are contained in $S$, it means that the path is a straight line without any branch, and so the topological space $\left(S, \mathcal{T}_{S}\right)$ consists of basic elements is a 1-manifold.

If $|S| \geq 1$ and $\forall p t \in S$,pt.ls = 1,pt.rs $=p t . d s$, $\forall t s \in S$,ts.ls $=1$,ts.rs $=$ ts.os, the overlapping path is also a straight line due to all the switches having the same position and all the track sections are connected and ready for generating movement authority. As a result, the topological space $\left(S, \mathcal{T}_{S}\right)$ is a 1 -manifold.

In what follows we prove that if the topological space $S$ is a 1-manifold, then the safety requirements are satisfied. In order to make it simple to prove, we prove the contra-positive statement, i.e. if the safety requirements defined in definition 19 are not satisfied, then the topological space $S$ would not be a 1-manifold.

If there are switch elements contained in $S$ and $\exists p t \in$ $S, p t . l s=1, p t . r s \neq p t . d s$, then it means that there exists an branch like shape (the topological space of the intersection path between $p_{1}$ and $p_{2}$ as shown in Fig. 6) in the intersection topological space $S$, i.e., $\exists \gamma \in p 1, \exists \gamma^{\prime} \in p 2$ and $\gamma \in S, \gamma^{\prime} \in$ $S, \operatorname{Pro1}(\gamma)=\operatorname{Pro}\left(\gamma^{\prime}\right)$ and $\operatorname{Pro} 2(\gamma) \neq \operatorname{Pro} 2\left(\gamma^{\prime}\right)$.

Based on the intuitive shape of switch and track sections as introduced above, the branch here is consisted of a track section $t s$ and a switch $p t$. They are connected at a point which is the end location of the track section ts.pr and also the stem location of the switch pt.pm. We denote the intersection point as $p$. Then $p$ has three directions to go, ts.pl (the left end location of $t s$ ), pt.pr (the reverse end location of $p t$ ) or $p t . p n$ (the normal end location of $p t$ ).

Suppose $S$ is a 1-manifold, then point $p \in S$ is locally Euclidean of dimension 1 at the point. Consequently, $p$ and its neighborhood $U$ is homeomorphic to an open ball $B:=$ $B(0, \epsilon) \subset \mathbb{R}^{n}$, where point $p$ is mapped to $0, n$ represents the dimension of the ball $B$. The homeomorphism $U \rightarrow B$ restricts to a homeomorphism $U-\{p\} \rightarrow B-\{0\}$ (here the minus operator indicated the exclusion of the set of points from the topological space). Now $B-\{0\}$ is either connected if $n \geq 2$ or has two connected components if $n=1$. Since
$U-\{p\}$ has three connected components, there can be no homeomorphism from $U-\{p\}$ to $B-\{0\}$. This contradiction proves that the branch like shape is not locally Euclidean at $p$, therefore the path $S$ is not a 1-manifold.

If the line condition is not satisfied, then the virtual coupling operation should not be allowed. At this time, the movement authority of the following train would be as far as the stem location of the switch (the switch with different required positions by two paths). For example, if the intersection set of two paths is same to the "shape" as shown in Fig 6, i.e., the following train has a different required position of a switch with the preceding train, then the movement authority for the following train would be as far as the stem position of the switch, unless the switch position has changed to the required position of the following train.

\section{Movement authority computation of virtual coupling}

In this subsection, we would like to talk about the second problem mentioned in Section II-B on the basis of the line condition introduced in section III-C is satisfied. The curves below are defined in the topological space of the intersection set of paths.

We first introduce the dynamic curves of train movement and then give the definition of the movement authority of virtual coupling based on topological manifold theory.

As introduced in our previous paper [30],the movements of trains are defined as n-manifold denoted by $S$. Then we map the neighborhood $U \subseteq S$ into a location-speed $\left(\mathbb{R}^{2}\right.$, which is a 2-dimension) or location ( $\mathbb{R}^{1}$, which is 1-dimension), speed $\left(\mathbb{R}^{1}\right.$, which is 1 -dimension) Euclidean space through its corresponding coordinate map functions $\left(\xi_{s}, \xi_{i}\right)$ as shown in equation 15,21 and 25). Finally, we can compute the relevant variables according to specific charts (the pair of $U$ and its map function).

Considering the accuracy requirement for future virtual coupling logic, the accurate train behavior under worst condition should be taken into consideration when calculating the movement authority for virtually coupled trains. Consequently, we introduce a safe brake curve which starts with current state of a train and a safe brake curve to a target state. Both consider train behavior under potential traction cutoff and brake buildup process, which makes the behavior model preciser than introduced in [30] for safety concerns. In addition, the zero train length assumption of [30] is removed by considering the maximum safe front and minimum safe rear of trains, which making our model more realistic.

1) Train dynamic curves: In this section, we introduce three kinds of train dynamic behavior under different situations: the ideal brake curve, the safe brake curve which starts with current state and the safe brake curve to a target state.

First we introduce the ideal train braking behavior, which is defined as shown in definition 21 . The train would start brake immediately from its current speed and location.

Definition 21: The ideal brake curve of a train is a 1manifold $S_{i}$ which is defined by equation 13. $s$ and $v$ represent train location and speed variables of a function. $s_{c}^{r}$ and $v_{c}$ represent current train's minimum safe rear end location and 
TABLE I: Nomenclature of curves in manifold theory

\begin{tabular}{|c|c|c|c|}
\hline Notations & Description & Notations & Description \\
\hline$s_{t c f}^{f}$ & $\begin{array}{l}\text { The maximum safe front end location of train at the end } \\
\text { of traction cutoff process }\end{array}$ & $v_{t c f}$ & Train speed at the end of traction cutoff process \\
\hline$s_{b b u}^{f}$ & $\begin{array}{l}\text { The maximum safe front end location of train at the end } \\
\text { of brake buildup process }\end{array}$ & $v_{b b u}$ & Train speed at the end of brake buildup process \\
\hline$S_{i}$ & The 1-manifold ideal brake curve & $U_{i}$ & The coordinate neighborhood of $S_{i}$ \\
\hline$\xi_{i}$ & The coordinate map of $S_{i}$ & $S_{s}$ & $\begin{array}{l}\text { The 1-manifold safe brake curve which starts with the current } \\
\text { maximum safe front end location and speed of train }\end{array}$ \\
\hline$U_{s}$ & The coordinate neighborhood of $S_{s}$ & $\xi_{s}$ & The coordinate map of $S_{s}$ \\
\hline$\eta(s, v)$ & $\begin{array}{l}\text { Concrete expression of safe brake curve starting from } \\
\text { current maximum safe front end location and speed }\end{array}$ & $\operatorname{dis}(v)$ & $\begin{array}{l}\text { The extra distance operated during traction cutoff } \\
\text { and brake buildup processes }\end{array}$ \\
\hline$S_{e}$ & The 1-manifold safe brake curve to a target location and speed & $U_{e}$ & The coordinate neighborhood of $S_{e}$ \\
\hline$\xi_{e}$ & The coordinate map of $S_{e}$ & $\mathcal{M}_{m a}$ & The 2-manifold with boundary \\
\hline Int $\mathcal{M}_{m a}$ & $\begin{array}{l}\text { The movement authority of a following train, } \\
\text { which is the interior of } \mathcal{M}_{m a}\end{array}$ & $\partial \mathcal{M}_{m a}$ & The boundary of $\mathcal{M}_{m a}$ \\
\hline$U_{m a}$ & The coordinate neighborhood of $\mathcal{M}_{m a}$ & $\xi_{m a}$ & The coordinate map of $\mathcal{M}_{m a}$ \\
\hline$s_{p}$ & The location of preceding train & $v_{p}$ & The speed of preceding train \\
\hline$S_{1}$ & The 1-manifold maximum speed limitation curve & $S_{2}$ & The 1-manifold current following train's location limitation curve \\
\hline$S_{3}$ & The 1-manifold zero speed limitation curve & $S_{4}$ & The 1-manifold preceding train's speed limitation curve \\
\hline$U_{j}$ & The coordinate neighborhood of $S_{j}$ & $\xi_{j}$ & The coordinate map of $S_{j}$ \\
\hline $\operatorname{MinSRE} E_{f}$ & the minimum safe rear end location of following train & $\operatorname{MaxSFE} E_{f}$ & the maximum safe front end location of the following train \\
\hline $\operatorname{MinSRE} E_{p}$ & the minimum safe rear end location of preceding train & $\operatorname{Max} S F E_{p}$ & the maximum safe front end location of the preceding train \\
\hline
\end{tabular}

speed, which are specific values. The chart is represented by $\left(U_{i}, \xi_{i}\right)$, where $U_{i}$ and $\xi_{i}$ are defined by equation 14 and 15 .

$$
\begin{gathered}
S_{i}=\left\{(s, v) \in \mathbb{R}^{2} \mid s-s_{c}^{r}=\frac{1}{2 d e c} v^{2}\right\} \\
U_{i}=\left\{(s, v) \in \mathbb{R}^{2} \mid s-s_{c}^{r}=\frac{1}{2 \operatorname{dec}} v^{2}, 0<v<v_{c}\right\} \\
\xi_{i}: U_{i} \rightarrow \mathbb{R} \quad \xi_{i}(s, v)=s \in\left(s_{c}^{r}, s_{c}^{r}+\frac{1}{2 \operatorname{dec}} v_{c}^{2}\right)
\end{gathered}
$$

The ideal brake curve is used to calculate the ideal brake behavior of the preceding train, that's why a train's minimum safe rear end location $s_{c}^{r}$ and speed $v_{c}$ are used in the equations. According to the ideal brake curve, we could define the safe brake curve as starting with current location and speed. The safe brake behavior considers the worst case condition of the braking process - that is a train would first cutoff the traction power and then buildup the brake. During those two processes, a train could experience extra acceleration phases in the worst situation.

We first introduce the relevant location and speed functions during the above two processes as shown in equation 16. $s_{t c f}^{f}$ and $s_{b b u}^{f}$ represent the maximum safe front end locations of the train at the end of the traction cutoff and brake buildup processes. $v_{t c f}$ and $v_{b b u}$ represent the corresponding speed values at those locations. They are affected by several concrete parameters according to the train type. The traction cutoff and brake buildup time durations are denoted by $t_{1}$ and $t_{2}$ respectively. The acceleration rate during traction cutoff and brake buildup process is denoted by $a_{1}$ and $a_{2}$ respectively. $s$ and $v$ in equation 16 represent the location and speed parameters of a train.

$$
\begin{aligned}
& s_{t c f}^{f}(s, v)=s+v t_{1}+0.5 a_{1} t_{1}^{2} \\
& v_{t c f}(v)=v+a_{1} t_{1} \\
& s_{b b u}^{f}(s, v)=s_{t c f}(s, v)+v_{t c f}(v) t_{2}+0.5 a_{2} t_{2}^{2} \\
& v_{b b u}(v)=v_{t c f}(v)+a_{2} t_{2}
\end{aligned}
$$

The extra distance $\operatorname{dis}(v)$ covered by the train during these two processes could be computed based on equation 17 .

$$
\operatorname{dis}(v)=v t_{1}+\frac{1}{2} a_{1} t_{1}^{2}+v t_{2}+a_{1} t_{1} t_{2}+\frac{1}{2} a_{2} t_{2}^{2}
$$

Then, we introduce the safe brake curve starting with the current maximum safe front end location and speed of a train, as shown in definition 22. The traction cutoff and brake buildup processes will be taken into consideration.

Definition 22: The safe brake curve which starts with the current maximum safe front end location and speed of a train is a 1-manifold $S_{s}$ that is defined by the equation 18 , where $\eta(s, v)$ is shown in equation 19.

$$
S_{s}=\left\{(s, v) \in \mathbb{R}^{2} \mid \eta(s, v)=0\right\}
$$

$\eta(s, v)=\left\{\begin{array}{lll}s_{c}^{f}-s+\frac{1}{2 a_{1}}\left(v^{2}-v_{c}^{2}\right), & \text { if } \quad v_{c} \leq v<v_{t c f} \\ s_{t c f}^{f}-s+\frac{1}{2 a_{2}}\left(v^{2}-v_{t c f}^{2}\right), & \text { if } \quad v_{t c f} \leq v \leq v_{b b u} \\ s_{b b u}^{f}-s+\frac{1}{2 d e c}\left(v_{b b u}^{2}-v^{2}\right), & \text { if } \quad 0 \leq v<v_{b b u}\end{array}\right.$

In equation 19, $s$ and $v$ represent the location and speed of the train. $s_{c}^{f}$ and $v_{c}$ represent the train's current maximum safe front end location and speed. $s_{t c f}\left(s_{c}^{f}, v_{c}\right), v_{t c f}\left(v_{c}\right)$, $s_{b b u}^{f}\left(s_{c}^{f}, v_{c}\right)$ and $v_{b b u}\left(v_{c}\right)$ are denoted by $s_{t c f}^{f}, v_{t c f}, s_{b b u}^{f}$ and $v_{b b u}$ for simplicity.

The chart is represented by $\left(U_{s}, \xi_{s}\right)$, where $U_{s}$ and $\xi_{s}$ are defined by equation 20 and 21 . The details of the map function $\xi_{s}(s, v)$ can be found in equation 22 .

$$
U_{s}=\left\{(s, v) \in \mathbb{R}^{2} \mid \eta(s, v)=0, s_{c}^{f} \leq s \leq s_{b b u}^{f}+\frac{1}{2 d e c} v_{b b u}^{2}\right\}
$$

$$
\xi_{s}: U_{s} \rightarrow \mathbb{R} \quad \xi_{s}(s, v)=s \in\left(s_{c}^{f}, s_{b b u}^{f}+\frac{1}{2 d e c} v_{b b u}^{2}\right)
$$




$$
\xi_{s}(s, v)=\left\{\begin{array}{lll}
s_{c}^{f}+\frac{1}{2 a_{1}}\left(v^{2}-v_{c}^{2}\right), & \text { if } \quad v_{c} \leq v<v_{t c f} \\
s_{t c f}^{f}+\frac{1}{2 a_{2}}\left(v^{2}-v_{t c f}^{2}\right), & \text { if } \quad v_{t c f} \leq v \leq v_{b b u} \\
s_{b b u}^{f}+\frac{1}{2 d e c}\left(v_{b b u}^{2}-v^{2}\right), & \text { if } \quad 0 \leq v<v_{b b u}
\end{array}\right.
$$

In what follows we introduce another dynamic behavior of trains, the safe brake curve to a target location and speed.

Definition 23: The safe brake curve to a target location and speed of a train is a 1-manifold $S_{e}$ which is defined by equation 23. Distance and speed are represented by $s$ and $v$ respectively. $\operatorname{dis}(v)$ is the same as above. The target speed and target location of the train are represented by $v_{t}$ and $s_{t}$.

$$
S_{e}=\left\{(s, v) \in \mathbb{R}^{2} \mid s_{t}-s-\operatorname{dis}(v)=\frac{1}{2 \operatorname{dec}}\left(v^{2}-v_{t}^{2}\right)\right\}
$$

The chart is represented by $\left(U_{e}, \xi_{e}\right)$, where $U_{e}$ and $\xi_{e}$ are defined by equation 24 and 25 . The maximum speed on the line is denoted by $m v$.

$$
\begin{aligned}
& U_{e}=\left\{(s, v) \in \mathbb{R}^{2} \mid s_{t}-s-\operatorname{dis}(v)=\right. \\
& \left.\frac{1}{2 \operatorname{dec}}\left(v^{2}-v_{t}^{2}\right), v_{t}<v<m v\right\} \\
& \xi_{e}: U_{e} \rightarrow \mathbb{R} \\
& \xi_{e}(s, v)=s \in\left(s_{t}-\operatorname{dis}(m v)-\frac{1}{2 \operatorname{dec}}\left(m v^{2}-v_{t}^{2}\right),\right. \\
& \left.s_{t}-\operatorname{dis}\left(v_{t}\right)\right)
\end{aligned}
$$

\section{2) Movement authority of a following train:}

Definition 24: The movement authority of a following train under virtual coupling is the interior of a 2-manifold with boundary which is denoted by $\operatorname{Int}_{m a}$. It is computed according to 2-manifold with boundary $\mathcal{M}_{m a}$, which is defined by equation 26 . The chart is represented by $\left(U_{m a}, \xi_{m a}\right)$, where $U_{m a}$ and $\xi_{m a}$ are defined by equation 27 and 28 .

$$
\begin{aligned}
\mathcal{M}_{m a}= & \left\{(s, v) \in \mathbb{R}^{2} \mid \max \left\{s_{f_{c}}^{f}, \xi_{s f}\left(s_{f}, v_{f}\right)\right\} \leq s \leq s_{p},\right. \\
& \left.\max \left\{v_{f}, 0\right\} \leq v \leq \min \left\{\operatorname{mv}, v_{p}\right\}\right\}
\end{aligned}
$$

$$
\begin{aligned}
U_{m a}= & \left\{(s, v) \in \mathbb{R}^{2} \mid \max \left\{s_{f c}^{f}, \xi_{s f}\left(s_{f}, v_{f}\right)\right\}<s<s_{p},\right. \\
& \max \left\{v_{f}, 0\right\}<v<\min \left\{m v, v_{p}\right\}, \\
& \left.0 \leq v_{f}<v_{f c}, 0 \leq v_{p} \leq \max \left\{v_{p c}, v_{f c}\right\}\right\} \\
& \xi_{m a}: U_{m a} \rightarrow \mathbb{R}^{2} \quad \xi_{m a}(s, v)=(s, v)
\end{aligned}
$$

The current maximum safe front end location and speed of a following train are represented by $\left(s_{f_{c}}^{f}, v_{f c}\right)$. The current minimum safe rear end location and speed of a preceding train are represented by $\left(s_{p c}^{r}, v_{p c}\right)$ respectively. The corresponding location and speed domains of a following and a preceding train are represented by $\left(s_{f}, v_{f}\right)$ and $\left(s_{p}, v_{p}\right)$. Take $s_{f c}^{f}$ and $s_{f}$ as an example, $s_{f c}^{f}$ is a specific location value of following train while $s_{f}$ is a set of possible location values of following train. $s_{p}$ is mapped according to different speed values (see equation 29). $\xi_{s f}\left(s_{f}, v_{f}\right)$ represents the coordinate function of following train, which maps $\left(s_{f}, v_{f}\right)$ to $s_{f}$.

$$
s_{p}=\left\{\begin{array}{cl}
\xi_{e f}\left(s_{p}, v_{p}\right), & \text { if } \quad v_{p c} \leq v_{p} \leq m v \\
\xi_{i p}\left(s_{p}, v_{p}\right), & \text { if } \quad 0 \leq v_{p}<v_{p c}
\end{array}\right.
$$

$U_{m a}$ is shown in Fig. 7. There are two trains running on the railway line with the same direction. The current maximum safe front end location and speed of the following train (the orange train) is denoted by $\left(s_{f c}^{f}, v_{f c}\right)$. The current minimum safe rear end location and speed of the preceding train (the blue train) is denoted by $\left(s_{p c}^{r}, v_{p c}\right)$. According to the kinds of train dynamic curves above, we could compute the safe brake curve denoted by $U_{s}$ which starts from $\left(s_{f_{c}}^{f}, v_{f_{c}}\right)$ of the following train. The safe brake curve to target $\left(s_{p c}^{r}, v_{p c}\right)$ of the following train is denoted by $U_{e}$. The ideal brake curve of the preceding train is denoted by $U_{i}$.

In order to acquire the movement authority, there are some static curves computed based on the movement authority of the following train. The curves are all 1-manifolds and denoted by $S_{1}, S_{2}, S_{3}$ and $S_{4}$. The corresponding charts of those curves are denoted by $\left(U_{1}, \xi_{1}\right),\left(U_{2}, \xi_{2}\right),\left(U_{3}, \xi_{3}\right)$ and $\left(U_{4}, \xi_{4}\right)$, where $U_{1}=\left\{(s, m v) \mid s_{f c}^{f}<s<\xi_{e f}(s, m v)\right\}$, $\left.\xi_{1}(s, v)=s, U_{2}=\left\{\left(s_{f_{c}}^{f}, v\right) \mid v_{f c}<v<m v\right)\right\}, \xi_{2}(s, v)=v$, $U_{3}=\left\{(s, 0) \mid \xi_{s f}(s, 0)<s<\xi_{i p}(s, 0)\right\}, \xi_{3}(s, v)=s$ and $U_{4}=\left\{\left(s, v_{p c}\right) \mid s_{p c}^{r}-\operatorname{dis}\left(v_{p c}\right)<s<s_{p c}^{r}\right\}, \xi_{4}(s, v)=s$. As shown in Fig. 7, $U_{1}$ represents the maximum speed restriction of the line. $U_{2}$ represents current location limitation of the following train. $U_{3}$ represents the zero speed limitation of the following train. $U_{4}$ represents the current speed limitation of the preceding train. The orange rectangle on the distance axis represent the movement authority in traditional moving block systems. The EoA is nearly the minimum safe rear end location of the preceding train. As for the fixed block systems, the box would be even shorter than the moving block one according to the location of the occupied track section by the preceding train.

The boundary $\partial \mathcal{M}_{m a}$ of $\mathcal{M}_{m a}$ is a 1-manifold defined by the atlas denoted by $U_{u}=\left\{U_{1} \cup U_{2} \cup U_{3} \cup U_{4} \cup U_{e} \cup U_{i} \cup U_{s}\right\}$. The movement authority of the following train is the interior Int $\mathcal{M}_{m a}$ of the 2-manifold with boundary $\mathcal{M}_{m a}$.

We can therefore compute the movement authority of a following train according to a preceding train's behavior, if the line condition on virtual coupling as shown in definition 20 is satisfied. If the condition is not satisfied, then the following train would take the last switch equipment which satisfied the line condition in the intersection topological space $S$ as the final unit. At this time, we could regard the last switch equipment as a preceding train whose location is the switch location and speed is zero. Then, the $s_{p}$ would change to the safe brake curve $\xi_{e f}\left(s_{p}, v_{p}\right)$ which takes the start location of the last switch unit as the End of the Authority (EoA). That would be the same protection logic as used by current train control systems which ensures the train stops before its EoA.

As for the maximum acceleration curve introduced in [30], we replace it with two static curves (the maximum speed limitation and the current location limitation of train's maximum safe front) instead. The acceleration curve could indeed assess train's traction ability with accurate maximum traction behavior, but has no effect on the safety side. In 


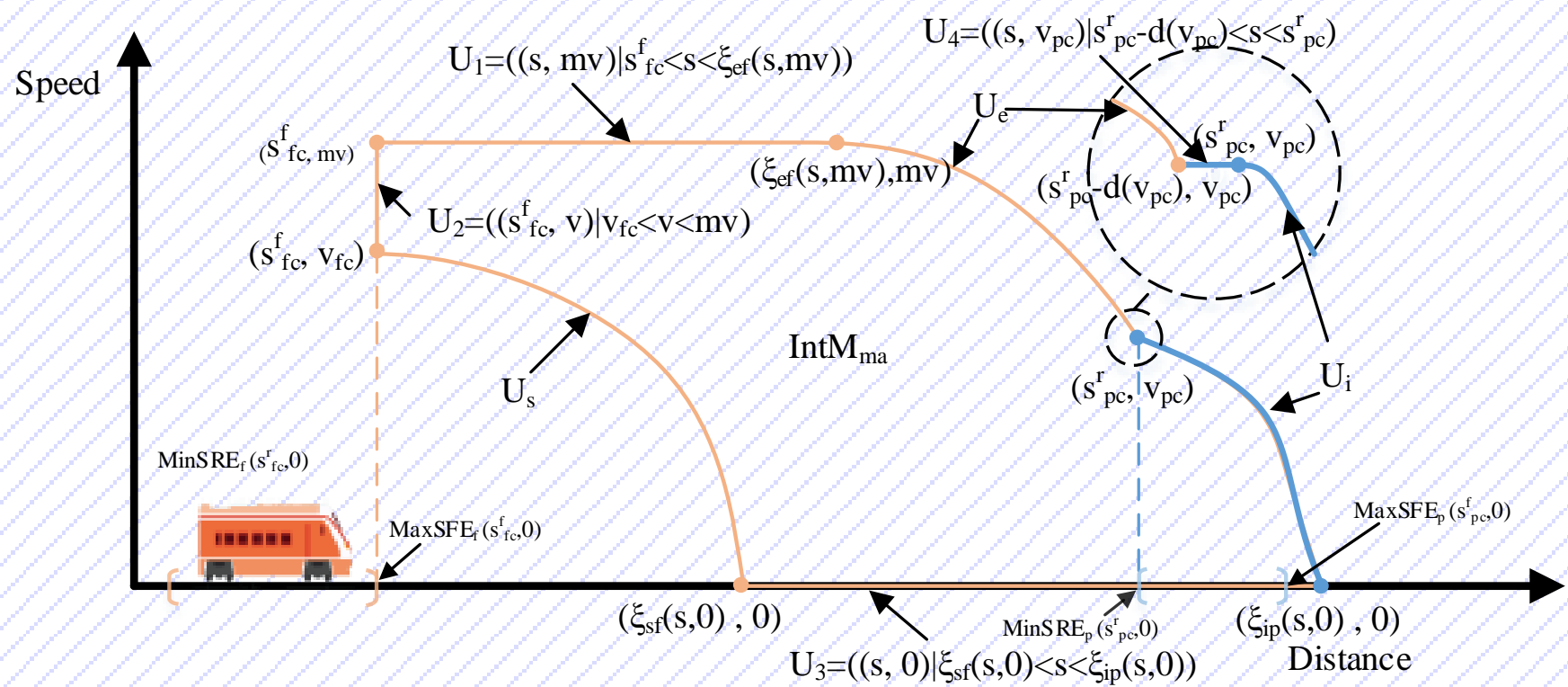

Fig. 7: The movement authority under virtual coupling represented by the manifold $\operatorname{Int} \mathcal{M}_{m a}$ of the following train $\left(\operatorname{MinSRE}_{f}\right.$, $M a x S F E_{f}, \operatorname{MinSRE} E_{p}, \operatorname{Max} S F E_{p}$ represent the minimum safe rear end and maximum safe front end locations of following and preceding trains )

addition, as for virtual coupling logic, more information on the movement dynamics of the interested trains is needed, which means that more information exchange is demanded to train-to-train communication. That's why we modeled the movement authority with $U_{2}$ and $U_{1}$ instead. In what follows, we would prove our proposed protection logic is safe by proving several theorems.

\section{E. Safety theorems for our virtual coupling}

In this part, 3 theorems are proven to interpret and verify the safety requirements of the virtual coupling control based on topological manifold theory. The safety requirements of virtual coupling are formally shown in definition 25 and 26. In what follows, the relation between the safety requirements and the manifold based description of the requirements is proved, which could be used to verify the safety of virtual coupling logic or further deduce work based upon the manifold theory.

Theorem 2 is about the safety of the movement authority of a following train. Here, safety means that the topological space of the movement authority is enough to stop the following train in the worst condition, without colliding with the preceding train based on the virtual coupling logic. Formally, the safety requirement for that is shown in definition 25 . It means the following train's location on its safe brake curve starting from the current location and speed would always be smaller than the preceding train's location on its ideal brake curve.

Definition 25: The safety requirement for the MA is: if $v_{f c} \geq v_{p c}$, then $\forall v \in\left(0, v_{p c}\right], \xi_{s f}\left(s_{f}, v_{f}\right)<\xi_{i p}\left(s_{p}, v_{p}\right)$. If $v_{f c}<v_{p c}$, then the requirement would be $\forall v \in$ $\left(0, v_{f c}\right), \xi_{s f}\left(s_{f}, v_{f}\right)<\xi_{i_{p}}\left(s_{p}, v_{p}\right)$.

Actually, a point on the boundary of the $\partial \mathcal{M}_{m a}$ represents any point between the final locations between the following and preceding trains. It represents the final location after specific behavior of trains. Thus, we consider the point $p \in U_{3}$ on the boundary of the manifold $\partial \mathcal{M}_{m a}$ as the condition.

Theorem 2: The movement authority Int $\mathcal{M}_{m a}$ of the following train is safe, if and only if there is a point $p \in U_{3}$ on the boundary of the manifold $\partial \mathcal{M}_{m a}$.

Proof 2: Suppose that there is no point $p \in U_{3}$ on the boundary of $\partial \mathcal{M}_{m a}$, then it means that $U_{3}$ is empty $\left(U_{3}=\right.$ $\left\{(s, 0) \mid \xi_{s f}(s, 0)<s<\xi_{i_{p}}(s, 0)\right\}$ according to the definition of $U_{m a}$ as shown in equation 27). As a result, $s$ has no valid values and the equation $\xi_{s f}(s, 0)<s<\xi_{i p}(s, 0)$ does not hold, which indicates that $\xi_{s f}\left(s_{f}, 0\right) \geq \xi_{i p}\left(s_{p}, 0\right)$. Hence, it contradicts the safety requirements in definition 25 .

If $\mathcal{M}_{m a}$ of the following train is safe, then $\xi_{s f}(s, 0)<$ $\xi_{i p}(s, 0)$ and $U_{3}$ have valid values. In what follows we need to prove that there is a point $p \in U_{3}$ that is a boundary point of $\partial \mathcal{M}_{m a}$, not in the interior Int $\mathcal{M}_{m a}$. According to the definition of coordinate neighborhood $U_{3}\left\{(s, 0) \mid \xi_{s f}(s, 0)<\right.$ $\left.s<\xi_{i p}(s, 0)\right\}, p \in U_{3}$ is homeomorphic to an open subset of $\partial \mathbb{H}^{2}=\left\{(s, v) \in \mathbb{R}^{2} \mid v=0\right\}$. Hence, point $p$ is on the boundary $\partial \mathcal{M}_{m a}$ of $\mathcal{M}_{m a}$.

Based on theorem 2, the safety of the movement authority under virtual coupling logic can be guaranteed.

Theorem 3 could be used to guarantee the safety of the following train's behavior under the virtual coupling control. We assume the train would follow the safe brake curve starting with its current location and speed. The safety requirement is that the behavior of the following train represented by its location and speed would be contained in the MA as shown in definition 26 . The boundary $\partial \mathcal{M}_{m a}$ represents the unsafe area under the worst condition during train operation.

Definition 26: Suppose the behavior of a following train is a 1-manifold $\mathcal{M}_{f}$. The safety requirement for the following train behavior is that $\forall p \in \mathcal{M}_{f}, p$ is not in the boundary 
$\partial \mathcal{M}_{m a}$.

Theorem 3: The condition $\forall p \in \mathcal{M}_{f}, p \in$ Int $_{\text {ma }}$ in the topological view implies the safety requirement of the following train behavior.

Proof 3: Under the worst situation assumption, a preceding train would be the one described by $S_{i}$ and a following train would obey the safe brake action described as $S_{s}$. Let point $p \in$ Int $_{m a} \subset \mathcal{M}_{m a}$ and $p \in$ Int $_{m a} \cap \partial \mathcal{M}_{m a}$. Suppose $V$ containing $p$ is an open subset of Int $\mathcal{M}_{m a}$ and it is homeomorphic to an open subset of $\mathbb{R}^{2}$. Clearly for point $p$ there is a neighborhood $U \subset$ Int $\mathcal{M}_{m a}$ of $p$ that is homeomorphic to the open ball $B^{2}\left(B^{2}\right.$ is a subset of $\mathbb{R}^{2}$ ). Now $U$ is also homeomorphic to an open interval $I$ ( $I$ is a subset of $\mathbb{R}$ ), since $p \in \partial \mathcal{M}_{m a}$ and $\partial \mathcal{M}_{m a}$ is a 1manifold. However, $I$ is not homeomorphic to $B^{2}$. Hence, point $p \in \operatorname{Int} \mathcal{M}_{m a}$ is disjoint with the boundary $\partial \mathcal{M}_{m a}$. The train behavior is safe.

Theorem 4: The MAs represented by the 2-manifold for different trains in the virtual coupling queue do not intersect with each other.

Proof 4: Suppose that we have three trains $t 1, t 2, t 3$ in the same direction on the same part of a railway line. We assume train $t 3$ is the preceding train of the three, and is followed by $t 2$, then $t 1$. The following two trains $t 1$ and $t 2$ are under control of the virtual coupling logic. In the virtual coupling logic, the MA of $t 1$ represented by the interior of 2-manifold Int $\mathcal{M}_{m a 1}$ would be computed according to the movements of $t 2$ as its preceding train as introduced above. Similarly for train $t 2$, which computes its MA Int $\mathcal{M}_{m a 2}$ based on the movement of $t 3$ serving as the preceding train.

The atlas for the whole virtually coupled trains is the union of each denoted by $U_{m a}=U_{m a 1} \cup U_{m a 2}$ as we defined above. We denote the train $t 2$ as a point $p\left(s_{2}, v_{2}\right)$. According to the definition of MA, the safe brake curve $S_{e}$ of $p\left(s_{2}, v_{2}\right)$ composed of the boundary $\partial \mathcal{M}_{m a 2}$ and the ideal brake curve $S_{i}$ composed of the boundary $\partial \mathcal{M}_{m a 1}$ do not intersect with each other except for the point $t 2$, because of the extra brake behavior in the safe brake behavior. The point $p\left(s_{2}, v_{2}\right)$ is the intersection point between the safe brake curve of $t 2$ represented by $\xi_{s}\left(s_{2}, v_{2}\right)$ and the ideal brake behavior of $t 2$ represented by $\xi_{i}\left(s_{2}, v_{2}\right)$, which is $t 2=p\left(s_{2}, v_{2}\right)$ itself. Now we need to prove that the intersection point $p\left(s_{2}, v_{2}\right)$ does not belong to any of the MA of $t 2$ or $t 1$.

Since point $p\left(s_{2}, v_{2}\right)$ belongs to both boundaries of $\mathcal{M}_{m a 1}$ and $\mathcal{M}_{m a 2}$, i.e. $p \in \partial \mathcal{M}_{m a 1} \cap \partial \mathcal{M}_{m a 2}$, as proved in theorem 3, $p\left(s_{2}, v_{2}\right)$ is not in Int $\mathcal{M}_{m a 1}$ and Int $\mathcal{M}_{m a 2}$. As a result, the MAs for different trains in the virtual coupling queue are separated from each other.

Theorem 4 could be used to guarantee the safety of the movement authorities of both the following and preceding trains.

\section{CASE STUDY}

In this section, we undertake a case study in order to illustrate the proposed method based on engineering data from a real railway line. The model is built with current train control systems equipment, which contains track sections.

\section{A. Example Data and Scenario}

For the case study, we will use data from an existing railway track plan (see Fig. 8). The departure signal of the railway line serves as the stop location in the model. There are three trains on the railway line. Train 1 is scheduled to run from its current location to station LYN. The scheduled stop location in LYN station is the location of the existing signal X3. Train 2 is running from its current location to signal XI in station LYN. Train 3 is scheduled to stop before the signal X5 in station LYN.

The topological paths $p_{T L}$ and $p_{T T}$ for Train 1 and Train 2 are shown in Fig. 9. Train 1, Train 2 and Train 3 are represented by $u_{1}, u_{22}$ and $u_{16}$. Switches are represented by green topological elements, the stop locations are represented by yellow topological elements and the track sections are represented by purple topological elements. The dashed and dotted lines represent the $p_{T L}$ of Train 1 and Train 2 , denoted by $p_{T L 1}$ and $p_{T L 2}$. The solid lines represent the $p_{T T}$ of Train 1 and Train 2 , denoted by $p_{T T 1}$ and $p_{T T 2}$. The path for Train 1 is in orange and path for Train 2 is in blue. As we can see from Fig. 9, $p_{T L 1}=$ $\left\langle\gamma_{1}, \gamma_{2}, \gamma_{3}, \gamma_{5}, \gamma_{19}, \gamma_{20}, \gamma_{21}, \gamma_{23}, \gamma_{24}, \gamma_{22}, \gamma_{25}, \ldots, \gamma_{29}, \gamma_{7}, \gamma_{9}\right.$, $\left.\gamma_{11}, \gamma_{13}, \gamma_{15}, \gamma_{10}, \gamma_{12}\right\rangle, p_{T T 1}=\left\langle\gamma_{1}, \gamma_{2}, \gamma_{3}, \gamma_{5}, \gamma_{19}, \gamma_{20}, \gamma_{21}\right.$, $\left.\gamma_{23}, \gamma_{24}, \gamma_{22}\right\rangle$. Hence, the intersection space $S_{1}$ between $p_{T L 1}$ and $p_{T T 1}$ is $S_{1}=\left\{\gamma_{1}, \gamma_{2}, \gamma_{3}, \gamma_{5}, \gamma_{19}, \gamma_{20}, \gamma_{21}, \gamma_{23}, \gamma_{24}, \gamma_{22}\right\}$. Since the required positions of switch units $u_{3}$ and $u_{5}$ by $p_{T L 1}$ and $p_{T T 1}$ are the same, the intersection set is a 1-manifold. In addition, since for all the track sections and switches in $S$, the value of the map function $\Lambda_{P}()$ and $\Lambda_{T}()$ is 1 , the intersection space $S_{1}$ can be used to realize the virtual coupling control. The results are also given in Table II.

$\begin{array}{ccr}\text { Regarding } p_{T L 2} \quad \text { and } p_{T T 2}, & p_{T L 2} & = \\ \left\langle\gamma_{22}, \gamma_{23}, \ldots, \gamma_{29}, \gamma_{7}, \gamma_{9}, \gamma_{11}, \gamma_{6}, \gamma_{8}\right\rangle \text { and } & p_{T T 2}= \\ \left\langle\gamma_{22}, \gamma_{23}, \ldots, \gamma_{29}, \gamma_{7}, \gamma_{9}, \gamma_{11}, \gamma_{13}, \gamma_{15}, \gamma_{14}, \gamma_{16}\right\rangle, & \text { the }\end{array}$ intersection space $S_{2}$ between $p_{T L 2}$ and $p_{T T 2}$ is $S_{2}=\left\{\gamma_{22}, \gamma_{23}, \ldots, \gamma_{29}, \gamma_{7}, \gamma_{9}, \gamma_{11}\right\}$. Since the required positions of switch 9 in station LYN are different, the intersection space $S_{2}$ is not a 1-manifold. The reason is that there are three components for the stem location of switch 9, which is $\gamma_{11}$ in Fig. 9. As a result, train 2 can only be allowed to run to the location before the stem location of switch 9 in station LYN, which is compatible with the notion of the traditional EoA of the MA.

\section{B. Simulations}

In order to show that our method can implement a safe virtual coupling train control on a single railway line, we conduct a simulation to demonstrate it. The line data and scenario are described as above. We implement virtual coupling safety protection control logic of two trains (Train 1 and Train 2) with $\mathrm{C}++$ code on two computers. Here the train-to-train communication is implemented through a connected net wire, so there is almost no delay on the communication part.

The parameters for the Train1, Train2 are the same due to the assumption that trains are of the same type. The emergency braking deceleration rate is $1 \mathrm{~m} / \mathrm{s}^{2}$. The values of traction cutoff and brake buildup time durations $t_{1}$ and $t_{2}$ are $0.2 \mathrm{~s}$ 


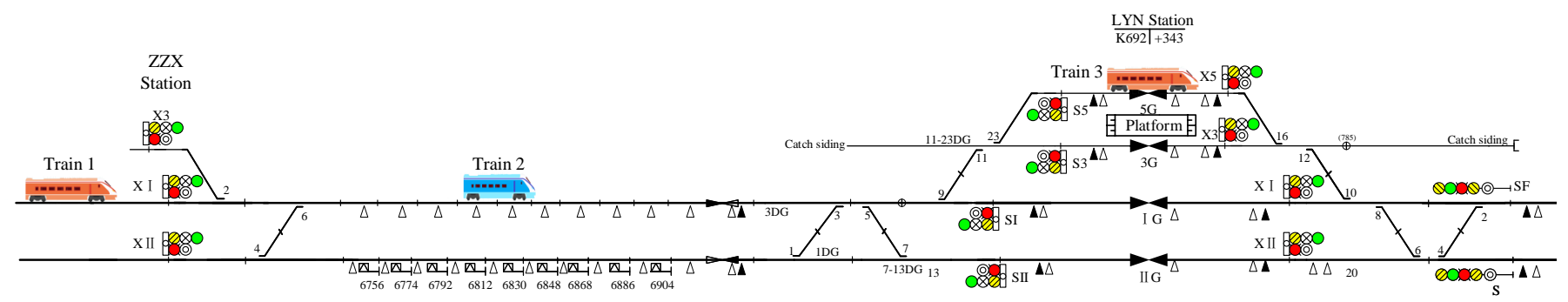

Fig. 8: Part of an existing railway line in China

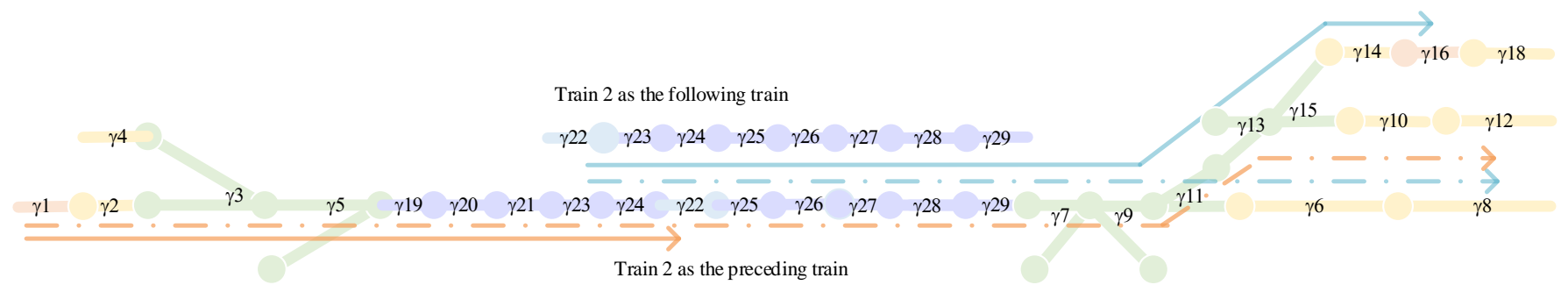

Fig. 9: The $p_{T T}$ and $p_{T L}$ in topological unit representation

TABLE II: Cases concerning whether an appraised train is allowed to be virtually coupled

\begin{tabular}{|c|c|c|c|c|c|c|c|}
\hline $\begin{array}{l}\text { Appraised } \\
\text { train }\end{array}$ & $\begin{array}{l}\text { Preceding } \\
\text { train }\end{array}$ & $p_{T L}$ & $p_{T T}$ & $\begin{array}{l}\text { Intersection } \\
\quad \text { set } S\end{array}$ & $\begin{array}{l}\text { Switches } \\
\text { in } S\end{array}$ & $\begin{array}{c}\text { Is } S \text { a } \\
\text { 1-manifold }\end{array}$ & $\begin{array}{l}\text { Virtually coupled } \\
\text { permission }\end{array}$ \\
\hline Train 1 & Train 2 & $\begin{array}{c}\left\langle\gamma_{1}, \gamma_{2}, \gamma_{3}, \gamma_{5}, \gamma_{19}, \gamma_{20}\right. \\
\gamma_{21}, \gamma_{23}, \gamma_{24}, \gamma_{22}, \gamma_{25} \\
\quad \ldots, \gamma_{29}, \gamma_{7}, \gamma_{9}, \gamma_{11} \\
\left.\quad \gamma_{13}, \gamma_{15}, \gamma_{10}, \gamma_{12}\right\rangle\end{array}$ & $\begin{array}{c}\left\langle\gamma_{1}, \gamma_{2}, \gamma_{3}, \gamma_{5}\right. \\
\gamma_{19}, \gamma_{20}, \gamma_{21} \\
\left.\gamma_{23}, \gamma_{24}, \gamma_{22}\right\rangle\end{array}$ & $\begin{array}{c}\left\{\gamma_{1}, \gamma_{2}, \gamma_{3}, \gamma_{5}\right. \\
\gamma_{19}, \gamma_{20}, \gamma_{21} \\
\left.\gamma_{23}, \gamma_{24}, \gamma_{22}\right\}\end{array}$ & $\gamma_{3}, \gamma_{5}$ & Yes & Yes \\
\hline Train 2 & Train 3 & $\begin{array}{c}\left\langle\gamma_{22}, \gamma_{23}, \ldots, \gamma_{29}, \gamma_{7}\right. \\
\left.\gamma_{9}, \gamma_{11}, \gamma_{6}, \gamma_{8}\right\rangle\end{array}$ & $\begin{array}{c}\left\langle\gamma_{22}, \gamma_{23}, \ldots, \gamma_{29}\right. \\
\gamma_{7}, \gamma_{9}, \gamma_{11}, \gamma_{13} \\
\left.\gamma_{15}, \gamma_{14}, \gamma_{16}\right\rangle\end{array}$ & $\begin{array}{l}\left\{\gamma_{22}, \gamma_{23}, \ldots, \gamma_{29}\right. \\
\left.\quad, \gamma_{7}, \gamma_{9}, \gamma_{11}\right\}\end{array}$ & $\gamma_{7}, \gamma_{9}, \gamma_{11}$ & No & No \\
\hline
\end{tabular}

and $1 s$ respectively for all trains in the example. The values of the possible acceleration during traction cutoff and brake buildup process are $1 \mathrm{~m} / \mathrm{s}^{2}$ and $0.5 \mathrm{~m} / \mathrm{s}^{2}$ respectively.

As one moment during the whole simulation, the minimum safe rear end location of Train 1 is on the fourth track section from ZZX station, where the specific location and speed is $7678 m$ (the location of signal XI in station ZZX is $0 m$ ) with speed of $270 \mathrm{~km} / \mathrm{h}$, Train 2 is running at $8503 \mathrm{~m}$ with its maximum speed $250 \mathrm{~km} / \mathrm{h}$. The movement authority of train 1 under virtual coupling at this moment is shown in Fig. 10, which is the interior area surrounded by of the orange curves.

If we assume that Train 1 is running at $6000 \mathrm{~m}$, then the traditional movement authority under fixed block principle would be the orange area on the distance axis. It starts from $6000 \mathrm{~m}$ to the border of the track section $(6686 \mathrm{~m})$, on which Train 2 is running. If the moving block principle is adopted, then the movement authority would be the gray area on the distance axis, which ends to $8483 \mathrm{~m}$, 20meters away from the minimum safe rear of Train 1.

As we can see in Fig. 10, the final stop location $\xi_{s f}(s, 0)$ - represented by the red diamond - of the following train is greater than the current location $s_{p c}$ - represented by the blue circle - of the preceding train, which cannot happen in a traditional moving block or fixed block signaling system. The simulation platform is a PC with 64-bit operating system, with

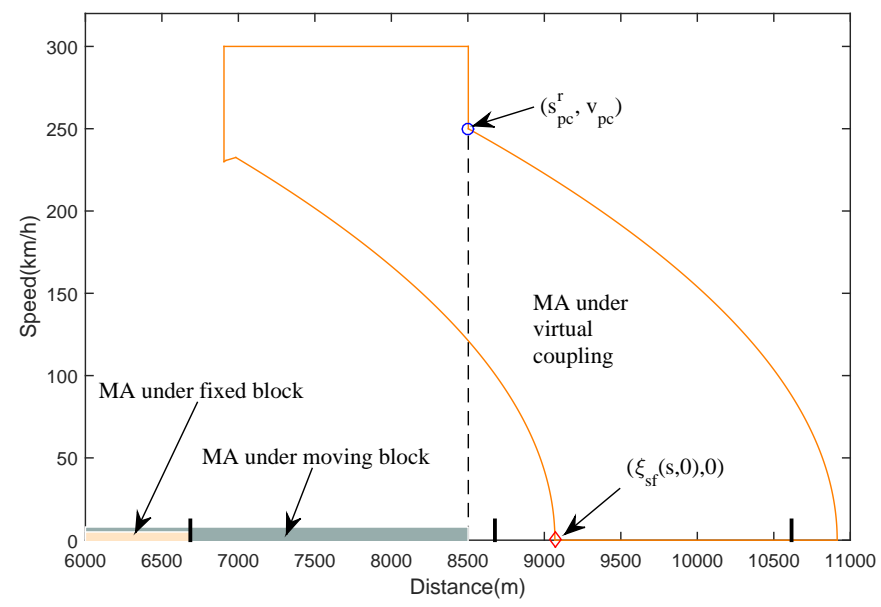

Fig. 10: Simulation scenario of virtual coupling control

an Intel i7-6700k and 32GB RAM. The simulation time for the computation of MA in our example is around $8 \mathrm{~ms}$.

\section{Discussion}

The results of the case study show that the proposed method for virtual coupling can compute the movement authority for any train. In reality the method can be used independently 
of concrete train types, which means that the parameters for the following train and preceding train could be different. The simulation time of the proposed manifold based safety protection control logic is also small enough to satisfy the real-time requirement of the train control system (here realtime requirement means the logic would take less computation time than the control cycle of the equipment). However, one should note that the time is only for calculation of the manifold protection logic, the time for other monitoring, diagnostic and communication loss is not considered.

Compared with other proposed modeling methods for train control system, which are based on the point-set topology, dynamic behavior of trains with their speed and location are taken into consideration and are represented by manifold definitions directly. The MA with consideration for continuous speed information could be computed for virtual coupling train control, which is impossible using current point-set topology based control logic. Compared with our previous method, the effect of switches and track sections are analyzed in detail under virtual coupling logic. The MA defined in this paper is more accurate since the effect of safe front and rear end locations of train are considered. Compared to the normal arithmetic based virtual coupling logic, railway signaling equipment information (such as switches) could also be taken into consideration under the same framework, which is essential for the safety protection logic of the virtual coupling system.

\section{Conclusions}

In this paper, a topological manifold based modeling method is proposed as a basis for protection logic of virtual coupling systems. We hope that the application of the proposed modeling and methodology will contribute to a higher level of integrity in the design and realization of railway virtual coupling control strategies. Essentially, the method provides a general formalism to model the concrete elements of train control systems. A safety analysis is presented with regards to the relevant locations of basic railway equipment. Based on that, a movement authority space for the virtual coupling control is described and implemented. This considers the dynamic behavior of trains in a combined way. Consequently, safety proofs of the system can be given in a straightforward manner with this method. By using this methodology, both basic railway signaling equipment control and dynamic train behavior could be formally described in a unified formalism, which has a significant advantage in the analyzing, designing and implementing of virtual coupling due to its mathematical nature.

The elements of railway networks under a virtual coupling logic are defined as topological units. The topological line condition for trains running safely under virtual coupling logic is clearly described by a theorem. The movement authority of a virtual coupling control system is given and combined with the dynamic train behavior curves, such that the traditional signaling principle to separate trains on the railway network can be preserved in an extended 2-dimensional speed and location space. The case study has shown that the proposed method is feasible for realizing the virtual coupling logic based on current train control system. It should result in fewer errors and less ambiguity in virtual coupling logic implementation, which is an essentially key problem for the pending technology. More complex scenarios of virtual coupling control have not been discussed in this paper, however this approach is expected to address complex situations in the future.

\section{ACKNOWLEDGMENT}

The authors would like to thank Mr. A. Walters for his contribution to the proofreading of the paper.

\section{REFERENCES}

[1] I. Mitchell, "ERTMS Level 4, Train Convoys or Virtual Coupling," IRSE International Technical Committee, Tech. Rep., 2016.

[2] Shift2Rail Joint Undertaking, "Amended annual work plan and budget for 2018," [Online]. Available: https://shift2rail.org/wp-content/uploads/ 2018/01/Ares-182422-Annex-1-S2R-1.pdf, Tech. Rep., 2018.

[3] H. Nakamura, "How to deal with revolutions in train control systems," Engineering, vol. 2, no. 3, pp. 380-386, 2016.

[4] T. Schumann, "Increase of capacity on the Shinkansen high-speed line using virtual coupling," International Journal of Transport Development and Integration, vol. 1, no. 4, pp. 666-676, 2017.

[5] H. Song and E. Schnieder, "Development and validation of a distance measurement system in metro lines," IEEE Transactions on Intelligent Transportation Systems, no. 99, pp. 1-16, 2018.

[6] T. Shen and H. Song, "A new movement authority based on vehiclecentric communication," Wireless Communications and Mobile Computing, vol. 2018, pp. 1-10, 2018.

[7] X. Wang, L. Liu, L. Zhu, and T. Tang, "Train-centric CBTC meets age of information in train-to-train communications," IEEE Transactions on Intelligent Transportation Systems, vol. 21, no. 10, pp. 4072-4085, 2020.

[8] J. Goikoetxea, "Roadmap towards the wireless virtual coupling of trains," in International Workshop on Communication Technologies for Vehicles. Springer, 2016, Lecture Notes in Computer Science, vol 9669, pp. 3-9.

[9] F. Flammini, S. Marrone, R. Nardone, A. Petrillo, S. Santini, and V. Vittorini, "Towards railway virtual coupling," in Proceedings of the 5th International Conference on Electrical Systems for Aircraft, Railway, Ship Propulsion and Road Vehicles (ESARS) and International Transportation Electrification Conference (ITEC), 2018.

[10] C. Di Meo, M. Di Vaio, F. Flammini, R. Nardone, S. Santini, and V. Vittorini, "ERTMS/ETCS virtual coupling: Proof of concept and numerical analysis," IEEE Transactions on Intelligent Transportation Systems, 2019.

[11] J. Felez, Y. Kim, and F. Borrelli, "A model predictive control approach for virtual coupling in railways," IEEE Transactions on Intelligent Transportation Systems, vol. 20, no. 7, pp. 2728-2739, 2019.

[12] "Railway applications-Communication, signalling and processing systems-Software for railway control and protection systems," European Committee for Electrotechnical Standardization (CENELEC), EN 50128, 2011.

[13] A. E. Haxthausen, An Introduction to Formal Methods for the Development of Safety-critical Applications. Technical University of Denmark, 2010.

[14] A. Ferrari, A. Fantechi, S. Gnesi, and G. Magnani, "Model-based development and formal methods in the railway industry," IEEE Software, vol. 30, no. 3, pp. 28-34, 2013.

[15] A. Fantechi, A. Ferrari, and S. Gnesi, "Formal methods and safety certification: challenges in the railways domain," in International Symposium on Leveraging Applications of Formal Methods. Springer, 2016, Lecture Notes in Computer Science, vol 9953, pp. 261-265.

[16] L. H. Vu, A. E. Haxthausen, and J. Peleska, "A domain-specific language for railway interlocking systems," in 10th Symposium on Formal Methods for Automation and Safety Railway and Automotive Systems, FORMS/FORMAT 2014. Technische Universität Braunschweig, 2014, pp. 200-209.

[17] L. H. Vu, A. E. Haxthausen, and J. Peleska, "Formal modelling and verification of interlocking systems featuring sequential release," Science of Computer Programming, vol. 133, pp. 91-115, 2017. 
[18] U. Berger, P. James, A. Lawrence, M. Roggenbach, and M. Seisenberger, "Verification of the european rail traffic management system in real-time Maude," Science of Computer Programming, vol. 154, pp. 61-88, 2018.

[19] P. James, F. Moller, H. N. Nguyen, M. Roggenbach, H. Treharne, and $\mathrm{X}$. Wang, "Ontrack: the railway verification toolset," in International Symposium on Leveraging Applications of Formal Methods. Springer, 2016, pp. 294-296.

[20] J. Peleska and W. Huang, "Test automation-foundations and applications of model-based testing," University of Bremen, lecture notes, available under http://www. cs. uni-bremen. de/agbs/jp/papers/test-automationhuang-peleska. pdf, 2017.

[21] C. Braunstein, A. E. Haxthausen, W. Huang, F. Hübner, J. Peleska, U. Schulze, and L. V. Hong, "Complete model-based equivalence class testing for the ETCS ceiling speed monitor," in International Conference on Formal Engineering Methods. Lecture Notes in Computer Science, vol 8829. Springer, 2014, pp. 380-395.

[22] D. Grasso, A. Fantechi, A. Ferrari, C. Becheri, and S. Bacherini, "Model based testing and abstract interpretation in the railway signaling context," in 2010 Third International Conference on Software Testing, Verification and Validation. IEEE, 2010, pp. 103-106.

[23] H. Wang, N. Zhao, B. Ning, T. Tang, and M. Chai, "Safety monitor for train-centric CBTC system," IET Intelligent Transport Systems, vol. 12, no. 8, pp. 931-938, 2018.

[24] L. H. Vu, A. E. Haxthausen, and J. Peleska, "A domain-specific language for generic interlocking models and their properties," in International Conference on Reliability, Safety and Security of Railway Systems. Springer, 2017, Lecture Notes in Computer Science, vol 10598, pp. 99115.

[25] P. James and M. Roggenbach, "Encapsulating formal methods within domain specific languages: A solution for verifying railway scheme plans," Mathematics in Computer Science, vol. 8, no. 1, pp. 11-38, 2014.

[26] H. Wang, F. Schmid, L. Chen, C. Roberts, and T. Xu, "A topologybased model for railway train control systems," IEEE Transactions on Intelligent Transportation Systems, vol. 14, no. 2, pp. 819-827, 2013.

[27] H. Wang, T. Xu, and T. Yuan, "Novel online safety observer for railway interlocking system," Journal of Transportation Engineering, vol. 139, no. 7, pp. 719-727, 2013.

[28] A. Platzer and J.-D. Quesel, "European train control system: A case study in formal verification," in International Conference on Formal Engineering Methods. Lecture Notes in Computer Science 5885. Springer, 2009, pp. 246-265.

[29] Y. Zhang, H. Wang, T. Yuan, J. Lv, and T. Xu, "Hybrid online safety observer for CTCS-3 train control system on-board equipment," IEEE Transactions on Intelligent Transportation Systems, no. 99, pp. 1-10, 2018.

[30] Y. Zhang and H. Wang, "Topological manifold-based monitoring method for train-centric virtual coupling control systems," IET Intelligent Transport Systems, vol. 14, no. 2, pp. 91-102, 2020.

[31] E. Quaglietta, M. Wang, and R. M. Goverde, "A multi-state trainfollowing model for the analysis of virtual coupling railway operations," Journal of Rail Transport Planning \& Management, vol. 15, p. 100195 , 2020, best Papers of RailNorrköping 2019. [Online]. Available: http://www.sciencedirect.com/science/article/pii/S2210970619300848

[32] H. Wang, T. Tang, C. Roberts, C. Gao, L. Chen, and F. Schmid, "A novel framework for supporting the design of moving block train control system schemes," Proceedings of the Institution of Mechanical Engineers, Part F: Journal of Rail and Rapid Transit, vol. 228, no. 7, pp. 784-793, 2014.

[33] W. T. Loring, An introduction to manifolds. Springer New York, 2008.

[34] J. Lee, Introduction to topological manifolds. Springer Science \& Business Media, 2010, vol. 202.

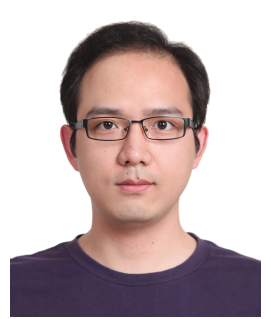

Yong Zhang received the B.Eng. degree in automation engineering from Chang'an University, Xian, China, in 2011, and the M.S. and Ph.D. degree from Beijing Jiaotong University, Beijing, China, in 2014 and 2020. He is currently doing postdoctoral research in Beihang University, Beijing, China.

His research interests are in fields of formal modeling and verification techniques, reliability analysis methods, intelligent optimization and scheduling for railway systems.

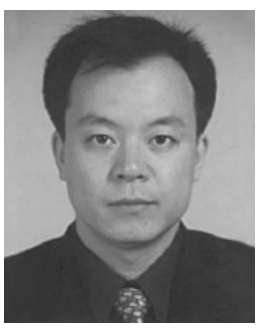

Haifeng Wang received the Ph.D. degree in system engineering from Beijing Jiaotong University, Beijing, China, in 2002.

$\mathrm{He}$ is currently a Professor of railway traffic information engineering and control with the National Engineering Research Center of Rail Transportation Operation and Control Systems, Beijing Jiaotong University. He has worked on computer-based interlocking systems and communications-based train control systems, mainly applied to main line railways and metro transit in China. In 2008, he was appointed as an expert of the Beijing metro transit signaling system's core technology R\&D and key demonstration projects. Since 2011, he has been a Visiting Researcher sponsored by the Chinese government with the Birmingham Centre for Railway Research and Education, University of Birmingham, Birmingham, U.K. His current research interests include software safety engineering methods for railway signaling systems, new architecture of train control systems, and methods for improving railway capacity.

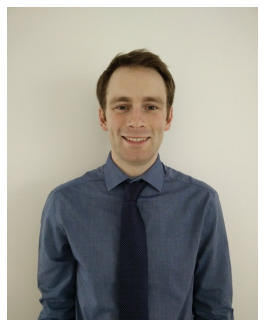

Phillip James is a Senior Lecturer in Computer Science at Swansea University. He completed his $\mathrm{PhD}$ in 2014 exploring the topic of verification of industrial control systems in 2014. His general research interests lie across the fields of formal methods, domain specific languages and technology transfer. He has over ten years experience in applying formal methods to railway signaling systems and has collaborated extensively with Siemens Rail Automation UK.

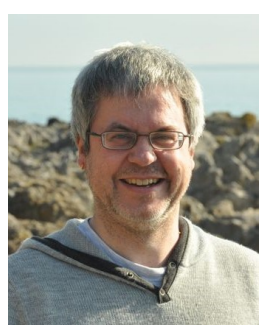

Markus Roggenbach holds a permanent position as a full Professor at Swansea University, where he built up his own research group "Processes and Data". He is a researcher in the area of safe and secure systems, covering safety as well as security. He works towards his vision of constructing safe and secure real world systems by applying formal methods. To this end, he often co-operates with industrial partners. His research agenda covers topics as varied as the verification of software designs, tool support for verification, specification languages, and testing, to name just a few.

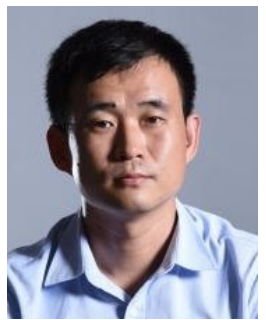

Daxin Tian (Senior Member, IEEE) received the $\mathrm{Ph}$.D. degree in computer science from Jilin University, Changchun, China, in 2007. He is currently a Professor with the School of Transportation Science and Engineering, Beihang University, Beijing, China. His research focuses on intelligent transportation systems, autonomous connected vehicles, swarm intelligence, and mobile computing. 